\title{
A Rigidity Theorem for Proper Holomorphic Mappings between Generalized Pseudoellipsoids
}

\author{
Atsushi HAYASHIMOTO \\ Nagano National College of Technology \\ (Communicated by K. Matsuzaki)
}

\begin{abstract}
Let $E(\alpha) \subset \mathbf{C}^{m+1}$ and $E(\beta) \subset \mathbf{C}^{n+1}$ be generalized pseudoellipsoids. Assume that the inequality $m<n$ holds. They are parametrized by $N$-tuples of positive integers $\alpha=\left(\alpha_{1}, \ldots, \alpha_{N}\right)$ and $\beta=\left(\beta_{1}, \ldots, \beta_{N}\right)$. (See introduction for the definition of a generalized pseudoellipsoid) Assume that there exists a proper holomorphic mapping between them. In this article, two facts are proved. Firstly, under the assumptions of the existence of such a mapping, certain nondegeneracy conditions of a submatrix of the Jacobian matrix and additional inequalities on dimensions, the parameters $\left(\alpha_{1}, \ldots, \alpha_{N}\right)$ and $\left(\beta_{1}, \ldots, \beta_{N}\right)$ coincide; $\alpha_{1}=\beta_{1}, \ldots, \alpha_{N}=\beta_{N}$ after re-ordering if necessary. Secondly, such a proper holomorphic mapping is a linear embedding up to automorphisms of a source and a target domains.
\end{abstract}

\section{Introduction}

Let $E\left(m ; m_{1}, \ldots, m_{N} ; \alpha_{1}, \ldots, \alpha_{N}\right)$ be a bounded domain in $\mathbf{C}^{m+1}$ with real analytic boundary defined by

$$
\begin{array}{r}
E\left(m ; m_{1}, \ldots, m_{N} ; \alpha_{1}, \ldots, \alpha_{N}\right)=\left\{\left(z, w_{1}, \ldots, w_{N}\right) \in \mathbf{C} \times \mathbf{C}^{m_{1}} \times \cdots \times \mathbf{C}^{m_{N}} ;\right. \\
\left.|z|^{2}+\left\|w_{1}\right\|^{2 \alpha_{1}}+\cdots+\left\|w_{N}\right\|^{2 \alpha_{N}}-1<0\right\}
\end{array}
$$

where $\alpha_{1}, \ldots, \alpha_{N} \in \mathbf{N}, \alpha_{1}, \ldots, \alpha_{N} \geq 2, m_{1}+\cdots+m_{N}=m$ and $\left\|w_{j}\right\|^{2 \alpha_{j}}=\left(\left|w_{j}^{1}\right|^{2}+\cdots+\right.$ $\left.\left|w_{j}^{m_{j}}\right|^{2}\right)^{\alpha_{j}}$ for $w_{j}=\left(w_{j}^{1}, \ldots, w_{j}^{m_{j}}\right) \in \mathbf{C}^{m_{j}}$. This is called a generalized pseudoellipsoid with $N$ blocks. See Remark 2 in $\S 2$ for the case of $\alpha_{1}=1$, namely there are some square norm terms. We say that $N$ is the number of the block of $w$. When we write $(\alpha)=\left(\alpha_{1}, \ldots, \alpha_{N}\right)$ and $(m)=\left(m_{1}, \ldots, m_{N}\right)$, we denote this domain by $E(m ;(m) ;(\alpha))$. It is biholomorphically equivalent to its unbounded representation

$$
\begin{gathered}
\Omega(m ;(m) ;(\alpha))=\left\{\left(z, w_{1}, \ldots, w_{N}\right) \in \mathbf{C} \times \mathbf{C}^{m_{1}} \times \cdots \times \mathbf{C}^{m_{N}} ;\right. \\
\left.\operatorname{Im} z>\left\|w_{1}\right\|^{2 \alpha_{1}}+\cdots+\left\|w_{N}\right\|^{2 \alpha_{N}}\right\}
\end{gathered}
$$

Received April 2, 2015; revised August 29, 2016

Mathematics Subject Classification: 32H35, 32V10

Key words and phrases: generalized pseudoellipsoid, proper holomorphic mapping, rigidity theorem 
via a mapping

$$
\begin{aligned}
\Psi_{1}: & \Omega(m ;(m) ;(\alpha)) \ni\left(z, w_{1}, \ldots, w_{N}\right) \\
& \mapsto\left(\frac{i-z}{i+z}, \frac{2^{1 / \alpha_{1}} w_{1}}{(i+z)^{1 / \alpha_{1}}}, \ldots, \frac{2^{1 / \alpha_{N}} w_{N}}{(i+z)^{1 / \alpha_{N}}}\right) \in E(m ;(m) ;(\alpha)) .
\end{aligned}
$$

Let $E(n ;(n) ;(\beta))$ be another generalized pseudoellipsoid. We denote by $\Omega(n ;(n) ;(\beta))$ the unbounded representation of $E(n ;(n) ;(\beta))$ via the mapping $\Psi_{2}$ defined in the same manner as in (2) and (3). Let $(\mathcal{F}, \mathcal{G})=\left(\mathcal{F}, \mathcal{G}_{1}, \ldots, \mathcal{G}_{N}\right)$ be a proper holomorphic mapping between $E(m ;(m) ;(\alpha))$ and $E(n ;(n) ;(\beta))$. Here, $\mathcal{F}\left(z, w_{1}, \ldots, w_{N}\right)$ is a $\mathbf{C}$-valued function and $\mathcal{G}_{j}\left(z, w_{1}, \ldots, w_{N}\right)$ is a mapping into the $j$-th block in $E(n ;(n) ;(\beta))$. Then we have a proper holomorphic mapping

$$
(F, G)=\Psi_{2}^{-1} \circ(\mathcal{F}, \mathcal{G}) \circ \Psi_{1}: \Omega(m ;(m) ;(\alpha)) \rightarrow \Omega(n ;(n) ;(\beta)) .
$$

We call the mapping $(F, G)=\left(F, G_{1}, \ldots, G_{N}\right)$ the unbounded representation of $(\mathcal{F}, \mathcal{G})$. We need the Jacobian matrix of $G_{k}=\left(G_{k}^{1}, \ldots, G_{k}^{n_{k}}\right)$

$$
\left(\begin{array}{cccc}
\frac{\partial G_{k}^{1}}{\partial z} & \frac{\partial G_{k}^{1}}{\partial w_{1}^{1}} & \cdots & \frac{\partial G_{k}^{1}}{\partial w_{N}^{m_{N}}} \\
\vdots & \vdots & \ddots & \vdots \\
\frac{\partial G_{k}^{n_{k}}}{\partial z} & \frac{\partial G_{k}^{n_{k}}}{\partial w_{1}^{1}} & \cdots & \frac{\partial G_{k}^{n_{k}}}{\partial w_{N}^{m_{N}}}
\end{array}\right)
$$

to state the main theorem. This is a submatrix of the Jacobian matrix of $(F, G)$ and we call this matrix the $k$-th block row in the Jacobian matrix of $(F, G)$. In the main theorem below, we give a certain condition on this matrix. See Remark 1 (3) bellow about this condition. For given domains $D_{1} \subset \mathbf{C}^{L}$ and $D_{2} \subset \mathbf{C}^{M}$, we say that a mapping $f: D_{1} \rightarrow D_{2}$ is equivalent to a mapping $g: D_{1} \rightarrow D_{2}$ if there exist automorphisms $\phi_{1}$ of $D_{1}$ and $\phi_{2}$ of $D_{2}$ such that $f=\phi_{2} \circ g \circ \phi_{1}$.

The following is the main theorem in this article.

MAIN THEOREM. Let $E(m ;(m) ;(\alpha))$ and $E(n ;(n) ;(\beta))$ be generalized pseudoellipsoids with $N$ blocks. Suppose that $2<m_{j}, 3<n_{j}$ and that $n-m<\min \left\{n_{1}, \ldots, n_{N}\right\}$. Let $\left(\mathcal{F}, \mathcal{G}_{1}, \ldots, \mathcal{G}_{N}\right): E(m ;(m) ;(\alpha)) \rightarrow E(n ;(n) ;(\beta))$ be a proper holomorphic mapping that is holomorphic up to the boundary. Assume that non-zero columns of any block row in the Jacobian matrix of the unbounded representation of $(\mathcal{F}, \mathcal{G})$ are linearly independent. Then we have the following:

(1) There exists a permutation $\sigma$ of $\{1, \ldots, N\}$ such that $\left.\mathcal{G}_{j}\right|_{w_{\sigma(j)}=0}=0$ for every $j$.

(2) If the permutation $\sigma$ in (1) satisfies the inequality $m_{\sigma(j)} \leq n_{j}<2 m_{\sigma(j)}-1$ for every $j$, then $\alpha_{\sigma(j)}=\beta_{j}$ for all $j$ and the proper holomorphic mapping $\left(\mathcal{F}, \mathcal{G}_{1}, \ldots, \mathcal{G}_{N}\right)$ is equivalent to $\left(\widetilde{\mathcal{F}}, \widetilde{\mathcal{G}}_{1}, \ldots, \widetilde{\mathcal{G}}_{N}\right)$ of the form

$$
\widetilde{\mathcal{F}}\left(z, w_{1}, \ldots, w_{N}\right)=z, \quad \widetilde{\mathcal{G}}_{j}\left(z, w_{1}, \ldots, w_{N}\right)=\left(w_{\sigma(j)}, 0\right), \quad 1 \leq j \leq N .
$$


REMARK 1. (1) The condition $n-m<\min \left\{n_{1}, \ldots, n_{N}\right\}$ is needed to prove that the preimage of the set of weakly pseudoconvex points is contained in the set of weakly pseudoconvex points. This condition may be omitted.

(2) The condition $m_{\sigma(j)} \leq n_{j}<2 m_{\sigma(j)}-1$ is needed to use a gap theorem for balls.

(3) About the assumption of the block row in the Jacobian matrix, we refer to the paper by X. Huang [4]. First he extends a proper holomorphic mapping between balls to their boundaries, and makes a composite function of the extended proper holomorphic mapping and a defining function of a target ball. The function is a defining function of a source ball. He applies CR vector fields and their complex conjugates one by one to the resulted defining function at a reference point. Then he obtains some linearly independent vectors which derived from once differentiation of the mapping. Those vectors are constituents of columns in the Jacobian matrix of the mapping under consideration. The reason why he can obtain the linearly independent vectors in this way is that all exponents in the defining function of a ball are two. Since exponents in the defining function of a generalized pseudoellipsoid are not necessarily two, we have no guarantee of obtaining the linearly independent vectors by once differentiation of the mapping, in general. Therefore we need to assume the existence of the linearly independent vectors in the Jacobian matrix.

The main theorem is a kind of a rigidity or a gap theorem. Typical gap theorem for balls is stated as follows. Let $F: B^{n} \rightarrow B^{N}$ be a proper holomorphic mapping which has certain regularity up to the boundary. Then there exist $k \in \mathbf{N}$ with $n \leq k \leq N$, an interval $I \subset \mathbf{N}$ and a proper holomorphic mapping $G: B^{n} \rightarrow B^{k}$ such that $F$ is equivalent to $(G, 0, \ldots, 0)$ provided that $N \in I$. If all $\alpha_{j}=1$, then $E(m ;(m) ;(1))$ is a ball, and this case is well understood. For example, S. Webster [9] proved that if a proper holomorphic mapping $f: B^{n} \rightarrow B^{n+1}, n>2$, extends to a $C^{3}$ mapping up to the boundary, then it is equivalent to a linear embedding. In the case of $n=2$, J. Faran [2] proved that, under the same assumption as in Webster's theorem, such a mapping is classified into four cases. J. Faran [3] also proved that any proper holomorphic mapping between $B^{n}$ and $B^{N}, n<N$, which is holomorphic up to the boundary is equivalent to the linear embedding given by $z \mapsto(z, 0)$ provided that $3<N<2 n-1$. X. Huang [4] obtained the same conclusion under the assumption that the mapping is only $C^{2}$ up to the boundary. If the codimension $N-n$ is higher, more is known. X. Huang, S. Ji, and D. Xu [5] proved that if a proper holomorphic mapping $F$ between $B^{n}$ and $B^{N}$ that is $C^{3}$ up to the boundary is equivalent to the mapping $z \mapsto\left(F_{\theta}(z), 0, \ldots, 0\right)$ provided that $4 \leq n \leq N \leq 3 n-4$, where $F_{\theta}(z)=$ $\left(z_{1}, \ldots, z_{n-1}, z_{n} \cos \theta, z_{1} z_{n} \sin \theta, \ldots, z_{n-1} z_{n} \sin \theta, z_{n}^{2} \sin \theta\right)$ for some $\theta$ with $0 \leq \theta \leq \pi / 2$. The paper by X. Huang, S. Ji and W. Yin [6] is a good survey of this topics.

The rigidity theorem for ellipsoids is obtained by P. Ebenfelt and D. N. Son in [1]. They studied the germs of holomorphic mappings at the origin between pseudoellipsoidal real hy- 
persurfaces in different dimensions

$$
\begin{aligned}
H:\left\{(z, w) \in \mathbf{C} \times \mathbf{C}^{n}:\right. & \left.\operatorname{Im} z=\sum_{j=1}^{n}\left|w_{j}\right|^{2 p_{j}}\right\} \\
& \rightarrow\left\{(z, w) \in \mathbf{C} \times \mathbf{C}^{N}: \operatorname{Im} z=\sum_{j=1}^{N}\left|w_{j}\right|^{2 q_{j}}\right\}
\end{aligned}
$$

and gave a classification of local holomorphic mappings between them under the condition of $N<2 n-1$. As far as the author knows, this is the only one rigidity theorem for pseudoellipsoids.

The organization of this article is the following. In $\S 2$, we pose some lemmas needed later. Main purpose of this section is to study the behavior of a proper holomorphic mapping on the weakly pseudoconvex set. In $\S 3$ and $\S 4$, we consider the generalized pseudoellipsoids with one block. In $\S 3$, we give a relation between the exponents which appear in the defining function. The ratio of the exponents $\alpha_{1}$ and $\beta_{1}$ in the source and the target defining functions respectively is an integer. In $\S 4$, we expand the components of the mapping and show that the ratio $\alpha_{1} / \beta_{1}$, which is an integer, is actually equal to one. In $\S 5$, we study the general case, that is, generalized pseudoellipsoids with $N$ blocks. In the last section, we obtain the general form of the proper holomorphic mapping $\left(\mathcal{F}, \mathcal{G}_{1}, \ldots, \mathcal{G}_{N}\right)$ between generalized pseudoellipsoids and compare it with Kodama's result [7]. Two conjectures are posed.

We use the following notation in this paper. Let $w=\left(w_{1}, \ldots, w_{N}\right) \in \mathbf{C}^{m_{1}} \times$ $\cdots \times \mathbf{C}^{m_{N}}, w_{j}=\left(w_{j}^{1}, \ldots, w_{j}^{m_{j}}\right)$ and $p=\left(p_{1}, \ldots, p_{N}\right) \in \mathbf{N}^{m_{1}} \times \cdots \times \mathbf{N}^{m_{N}}, p_{j}=$ $\left(p_{j}^{1}, \ldots, p_{j}^{m_{j}}\right), q_{j}=\left(q_{j}^{1}, \ldots, q_{j}^{m_{j}}\right) \in \mathbf{N}^{m_{j}}$, and $\alpha=\left(\alpha_{1}, \ldots, \alpha_{N}\right) \in \mathbf{N}^{N}$.

- The multi-index notation; $\left(w_{j}\right)^{p_{j}}=\left(w_{j}^{1}\right)^{p_{j}^{1}} \cdots\left(w_{j}^{m_{j}}\right)^{p_{j}^{m_{j}}}$ and $(w)^{p}=\left(w_{1}\right)^{p_{1}} \times$ $\cdots \times\left(w_{N}\right)^{p_{N}}$.

- The norms; $\left\|w_{j}\right\|^{2 \alpha_{j}}=\left(\left|w_{j}^{1}\right|^{2}+\cdots+\left|w_{j}^{m_{j}}\right|^{2}\right)^{\alpha_{j}},\left\||| w||^{2 \alpha}=\right\| w_{1} \|^{2 \alpha_{1}}+\cdots+$ $\left\|w_{N}\right\|^{2 \alpha_{N}}$ and $|\alpha|=\alpha_{1}+\cdots+\alpha_{N}$.

- The total degree; For a monomial $\left(w_{j}\right)^{p_{j}} \times\left(\bar{w}_{j}\right)^{q_{j}}$, the total degree of $w_{j}$ and $\bar{w}_{j}$ is the sum $\left|p_{j}\right|+\left|q_{j}\right|$.

- The variety; $\triangle^{j}=\left\{\left(z, w_{1}, \ldots, w_{N}\right) \in \mathbf{C} \times \mathbf{C}^{m_{1}} \times \cdots \times \mathbf{C}^{m_{N}} ; w_{j}=0\right\}$.

- $\operatorname{Re} z=x$

- $\partial \Omega$ is the boundary of $\Omega$.

We define an inner product $\langle w, z\rangle=w_{1} \bar{z}_{1}+\cdots+w_{k} \bar{z}_{k}$ for $\left(w_{1}, \ldots, w_{k}\right) \in \mathbf{C}^{k}$ and $\left(z_{1}, \ldots, z_{k}\right) \in \mathbf{C}^{k}$. When we need to distinguish the notation of source and target objects, we add 'tilde' on the target objects. 


\section{Image and preimage of weakly pseudoconvex set and expansions of mappings}

For simplicity, we use the notation $E(m ;(m) ;(\alpha))$ (resp. $E(n ;(n) ;(\beta)))$ instead of $E\left(m ; m_{1}, \ldots, m_{N} ; \alpha_{1}, \ldots, \alpha_{N}\right)$ (resp. $\left.E\left(n ; n_{1}, \ldots, n_{N} ; \beta_{1}, \ldots, \beta_{N}\right)\right)$. In order to classify the mapping $(\mathcal{F}, \mathcal{G})$ up to biholomorphic mappings, we classify its unbounded representation $(F, G)$.

LEMMA 2.1. If the $j$-th block $G_{j}$ in the unbounded representation $(F, G)$ vanishes on $w_{k}=0$, then $\mathcal{G}_{j}$ also vanishes there.

Proof. We restrict $\Psi_{2} \circ(F, G)(z, w)=(\mathcal{F}, \mathcal{G}) \circ \Psi_{1}(z, w)$ to $w_{k}=0$. Note that $\Psi_{1}$ and $\Psi_{2}$ have the forms (3). Pick up the $j$-th block components of $\Psi_{2}$ and $(\mathcal{F}, \mathcal{G})$ from the restricted equation. Then we get the result.

The Levi degenerate sets in $\partial \Omega(m ;(m) ;(\alpha))$ and $\partial \Omega(n ;(n) ;(\beta))$ are of the forms $\bigcup_{k=1}^{N}\left(\triangle^{k} \cap \partial \Omega(m ;(m) ;(\alpha))\right)$ and $\bigcup_{i=1}^{N}\left(\widetilde{\triangle}^{i} \cap \partial \Omega(n ;(n) ;(\beta))\right)$ respectively. For simplicity, we use the notation $\triangle^{k}$ instead of $\triangle^{k} \cap \partial \Omega(m ;(m) ;(\alpha))$.

Now we show that the preimage of the weakly pseudoconvex set is in the weakly pseudoconvex set. This is well known result for equi-dimensional case, but in general it does not hold. Let $\partial^{*} \Omega(m ;(m) ;(\alpha))$ and $\partial^{*} \Omega(n ;(n) ;(\beta))$ be the strictly pseudoconvex sets in $\partial \Omega(m ;(m) ;(\alpha))$ and in $\partial \Omega(n ;(n) ;(\beta))$ respectively.

LEMMA 2.2. Assume that the inequality $n-m<\min \left\{n_{1}, \ldots, n_{N}\right\}$ is fulfilled and that non-zero columns of any block row in the Jacobian matrix of $(F, G)$ are linearly independent. Then, for any $k(k=1, \ldots, N)$, there exists $j(j=1, \ldots, N)$ such that $(F, G)\left(\triangle^{j}\right) \subset \widetilde{\triangle}^{k}$.

ProOf. Let us denote

$$
\begin{aligned}
& Z=\{(z, w) \in \partial \Omega(m ;(m) ;(\alpha)) \mid \text { rank of the Jacobian matrix of }(F, G) \\
& \text { is not maximal at }(z, w)\} .
\end{aligned}
$$

We divide the proof into three parts.

CLAIM 1. Assume that the preimage under $(F, G)$ of the weakly pseudoconvex set is not empty. Then it is in the weakly pseudoconvex set.

To prove this, we show that any strictly pseudoconvex point is mapped to a strictly pseudoconvex point. Assume that there exists a strictly pseudoconvex point whose image is a weakly pseudoconvex point. We may assume that, after reordering, there exists $p \in \partial^{*} \Omega(m ;(m) ;(\alpha)) \backslash Z$ such that $(F, G)(p)=q \in \widetilde{\triangle}^{1} \cap \cdots \cap \widetilde{\triangle}^{k}$. The transposition of the Jacobian matrix of $(F, G)$ at $p$, which is denoted by $\left(F^{\prime}, G^{\prime}\right)^{t}(p)$, can be expressed as

$$
\left(F^{\prime}, G^{\prime}\right)^{t}(p)=R\left(\begin{array}{ll}
0 & E_{m+1}
\end{array}\right) V,
$$


where $R$ and $V$ are $(m+1) \times(m+1)$ and $(n+1) \times(n+1)$ nonsingular matrices respectively, $E_{m+1}$ is the $(m+1) \times(m+1)$ unit matrix. Let

$$
V=\left(\begin{array}{ll}
V_{1} & V_{2} \\
V_{3} & V_{4}
\end{array}\right)
$$

where $V_{1}$ is an $(n-m) \times 1$ matrix and $V_{4}$ is an $(m+1) \times n$ matrix. Set $\eta^{\prime}=$ $\left(\eta_{n-m+1}, \ldots, \eta_{n+1}\right) \in \mathbf{C}^{m+1}, \eta=\left(0, \ldots, 0, \eta^{\prime}\right) \in \mathbf{C}^{n+1}, \eta^{\prime} V_{3}=c_{0}$ and $\eta^{\prime} V_{4}=$ $\left(c_{1}, \ldots, c_{N}\right)$ for $c_{j} \in \mathbf{C}^{n_{j}}, j=1, \ldots, N$. Define $\xi$ as

$$
\xi=\eta\left(\begin{array}{c}
0 \\
E_{m+1}
\end{array}\right) R^{-1} \in \mathbf{C}^{m+1}
$$

and give conditions on $\xi$ to satisfy a system of equations:

$$
\left\{\begin{array}{l}
<\operatorname{grad}\left(-\operatorname{Im} F+\||| G||^{2 \beta}\right), \bar{\xi}>(p)=0, \\
c_{k+1}=0, \ldots, c_{N}=0
\end{array}\right.
$$

Here, differentials in gradient are with respect to the holomorphic differentials $\partial / \partial z, \partial / \partial w_{1}^{1}, \ldots, \partial / \partial w_{N}^{m_{N}}$. Then the vector $\xi \in \mathbf{C}^{m+1}$ satisfies $1+n_{k+1}+\cdots+n_{N}$ equations. By the assumption, we have $n-m<\min \left\{n_{1}, \ldots, n_{N}\right\}<n_{1}+\cdots+n_{k}$, which means that $1+n_{k+1}+\cdots+n_{N}=1+n-\left(n_{1}+\cdots+n_{k}\right)<m+1$. Therefore the system of equations (12) has non trivial solutions. Since $\partial \Omega(m ;(m) ;(\alpha))$ is strictly pseudoconvex at $p$, the Levi form of $-\operatorname{Im} z+\sum_{j=1, \ldots, N}\left\|w_{j}\right\|^{2 \alpha_{j}}$ at $p$ in the $\xi$-direction is strictly positive. In the following, we use the notation

$$
L_{j}(p)=\left(\begin{array}{ccc}
\frac{\partial^{2}\left\|w_{j}\right\|^{2 \alpha_{j}}}{\partial w_{j}^{1} \partial \bar{w}_{j}^{1}} & \cdots & \frac{\partial^{2}\left\|w_{j}\right\|^{2 \alpha_{j}}}{\partial w_{j}^{1} \partial \bar{w}_{j}^{m_{j}}} \\
\vdots & \ddots & \vdots \\
\frac{\partial^{2}\left\|w_{j}\right\|^{2 \alpha_{j}}}{\partial w_{j}^{m_{j}} \partial \bar{w}_{j}^{1}} & \cdots & \frac{\partial^{2}\left\|w_{j}\right\|^{2 \alpha_{j}}}{\partial w_{j}^{m_{j}} \partial \bar{w}_{j}^{m_{j}}}
\end{array}\right)(p)
$$

This is the notation for the source Levi form. When we treat the target Levi form, we use $\tilde{L}_{j}$. In this case, we change the exponents $\alpha_{j}$ to $\beta_{j}, m_{j}$ to $n_{j}$ and $w_{j}^{\lambda}$ to $\tilde{w}_{j}^{\lambda}$. Note that $-\operatorname{Im} F+\||| G||^{2 \beta}$ is a defining function of the source domain and that, therefore, the Levi form of it at $p$ in the $\xi$-direction must be positive. Since we have

$$
\xi\left(F^{\prime}, G^{\prime}\right)^{t}(p)=\left(\eta^{\prime} V_{3}, \eta^{\prime} V_{4}\right)=\left(c_{0}, c_{1}, \ldots, c_{k}, 0, \ldots, 0\right),
$$


the Levi form of $-\operatorname{Im} F+\||| G \mid\|^{2 \beta}$ at $p$ in the $\xi$-direction is calculated as

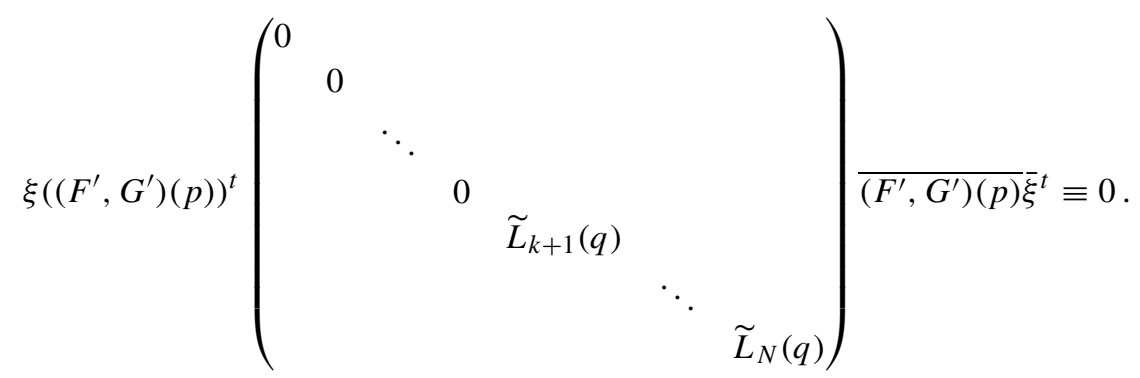

This contradicts the positivity. This proves Claim 1 .

Next, we shall show that the image of $\triangle^{j}$ is in only one $\widetilde{\Delta}^{k}$, not in two or more $\widetilde{\triangle}^{k}$,s.

CLAIM 2. There does not exist $j$ such that $(F, G)\left(\triangle^{j}\right) \subset \widetilde{\triangle}^{k_{1}} \cup \cdots \cup \widetilde{\triangle}^{k_{\ell}}$, $(F, G)\left(\triangle^{j}\right) \cap \widetilde{\triangle}^{k_{1}} \neq \emptyset, \ldots,(F, G)\left(\triangle^{j}\right) \cap \widetilde{\triangle}^{k_{\ell}} \neq \emptyset$ for some $\ell \geq 2$.

We show the case of $\ell=2$. The case of $\ell \geq 3$ is done by the same argument. Assume that there exists $j$ such that $(F, G)\left(\triangle^{j}\right) \subset \widetilde{\triangle}^{k_{1}} \cup \widetilde{\triangle}^{k_{2}},(F, G)\left(\triangle^{j}\right) \cap \widetilde{\triangle}^{k_{1}} \neq \emptyset$ and $(F, G)\left(\triangle^{j}\right) \cap$ $\widetilde{\triangle}^{k_{2}} \neq \emptyset$. Denote by $\triangle^{j,\left(k_{1}\right)}$ and $\triangle^{j,\left(k_{2}\right)}$ the subsets of $\triangle^{j}$ such that

$$
(F, G)\left(\triangle^{j,\left(k_{1}\right)}\right) \subset \widetilde{\triangle}^{k_{1}},(F, G)\left(\triangle^{j,\left(k_{2}\right)}\right) \subset \widetilde{\triangle}^{k_{2}} .
$$

Assume that $\left.G_{k_{2}}\right|_{\triangle^{j}} \not \equiv 0$. Then it follows from $\left.G_{k_{2}}\right|_{\triangle^{j,\left(k_{2}\right)}} \equiv 0$ that the inclusions

$$
\triangle^{j,\left(k_{2}\right)} \subset Z_{j}\left(G_{k_{2}}^{1}, \ldots, G_{k_{2}}^{n_{k_{2}}}\right) \subsetneq \triangle^{j}
$$

hold. Here, $Z_{j}\left(G_{k_{2}}^{1}, \ldots, G_{k_{2}}^{n_{k_{2}}}\right)$ is the set of common zeros of $G_{k_{2}}^{1}, \ldots, G_{k_{2}}^{n_{k_{2}}}$ in $\triangle^{j}$. These inclusions imply the following inequalities:

$$
\begin{aligned}
\operatorname{dim}_{\mathbf{R}} \triangle^{j,\left(k_{2}\right)} & \leq \operatorname{dim}_{\mathbf{R}} Z_{j}\left(G_{k_{2}}^{1}, \ldots, G_{k_{2}}^{n_{k_{2}}}\right) \\
& <\operatorname{dim}_{\mathbf{R}} \triangle^{j}\left(=\max \left\{\operatorname{dim}_{\mathbf{R}} \triangle^{j,\left(k_{1}\right)}, \operatorname{dim}_{\mathbf{R}} \triangle^{j,\left(k_{2}\right)}\right\}\right) .
\end{aligned}
$$

Thus we have

$$
\operatorname{dim}_{\mathbf{R}} \triangle^{j,\left(k_{2}\right)}<\operatorname{dim}_{\mathbf{R}} \triangle^{j}=\operatorname{dim}_{\mathbf{R}} \triangle^{j,\left(k_{1}\right)}
$$

and this implies that $\triangle^{j,\left(k_{2}\right)}$ is nowhere dense in $\Delta^{j}$. Since $\triangle^{j,\left(k_{1}\right)}$ is closed in $\triangle^{j}$, we have equalities

$$
\Delta^{j}=\overline{\triangle^{j} \backslash \triangle^{j,\left(k_{2}\right)}}=\overline{\triangle^{j,\left(k_{1}\right)}}=\triangle^{j,\left(k_{1}\right)} .
$$

Therefore we conclude that $\triangle^{j}=\triangle^{j,\left(k_{1}\right)}$, which implies $(F, G)\left(\triangle^{j}\right) \subset \widetilde{\triangle}^{k_{1}}$. This completes the proof of Claim 2.

Finally, we shall show that the preimage of any weakly pseudoconvex set is not empty. This may complete the proof of Lemma 2.2. 
CLAIM 3. There does not exist $k$ such that $(F, G)\left(\triangle^{1} \cup \cdots \cup \triangle^{N}\right) \cap \widetilde{\triangle}^{k}=\emptyset$.

Assume that there exists such $k$. Then we may assume that

$$
\begin{aligned}
& (F, G)\left(\triangle^{1} \cup \cdots \cup \triangle^{N}\right) \subset \widetilde{\triangle}^{1} \cup \cdots \cup \widetilde{\triangle}^{\ell}, \\
& (F, G)\left(\triangle^{1} \cup \cdots \cup \triangle^{N}\right) \cap\left(\widetilde{\triangle}^{\ell+1} \cup \cdots \cup \widetilde{\triangle}^{N}\right)=\emptyset .
\end{aligned}
$$

This means that there exist a point $Q=(x, 0, \ldots, 0) \in \partial \Omega(m ;(m) ;(\alpha))$ and a point $\widetilde{Q}=$ $\left(\tilde{z}, 0, \ldots, 0, \tilde{w}_{\ell+1}, \ldots, \tilde{w}_{N}\right) \in \partial \Omega(n ;(n) ;(\beta))$ such that $(F, G)(Q)=\widetilde{Q}$. Let $\xi$ be a CR vector at $Q$. The Levi form of $-\operatorname{Im} F+\||| G \mid\|^{2 \beta}$ at $Q$ in the $\xi$-direction is calculated as

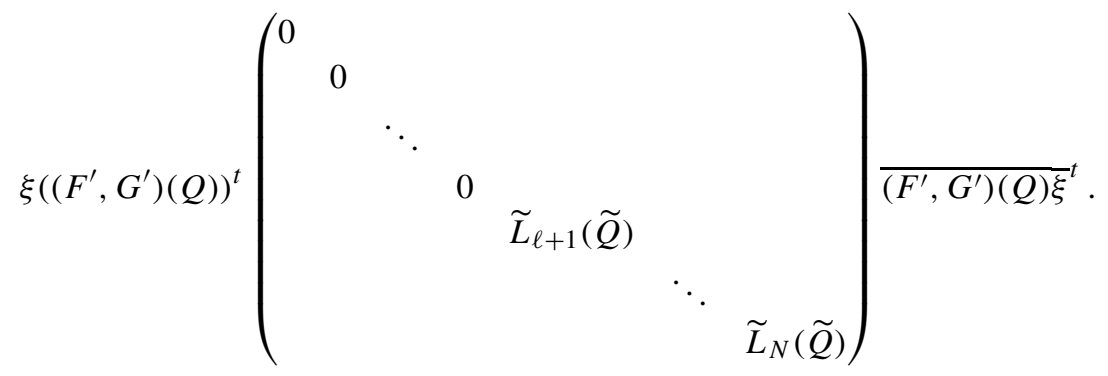

This is essentially the same as the Levi form of

$$
-\operatorname{Im} \tilde{z}+\sum_{j=\ell+1}^{N}\left\|\tilde{w}_{j}\right\|^{2 \beta_{j}}
$$

at $\left(\tilde{z}, \tilde{w}_{\ell+1}, \ldots, \tilde{w}_{N}\right)$. By assumption, for any $k(k=1, \ldots, N)$, non-zero columns in (5) are linearly independent. Thus, for any $\xi$, there exists at least one non-zero component from the $\left(n_{1}+\cdots+n_{\ell}+1\right)$-st component to the $\left(n_{1}+\cdots+n_{N}\right)$-th component in $\xi\left(\left(F^{\prime}, G^{\prime}\right)(Q)\right)^{t} \in$ $\mathbf{C}^{n+1}$. Therefore the Levi form (23) is positive. This is a contradiction. Therefore we conclude that there do not exist $Q$ and $\widetilde{Q}$, which proves Claim 3, and therefore Lemma 2.2 is proved.

The next lemma shows that the index $j$ appearing in Lemma 2.2 is unique.

LEMMA 2.3. Let $\left(F, G_{1}, \ldots, G_{N}\right): \Omega(m ;(m) ;(\alpha)) \rightarrow \Omega(n ;(n) ;(\beta))$ be a proper holomorphic mapping. Then there exists a permutation $\sigma$ of $\{1, \ldots, N\}$ such that $\left.G_{k}\right|_{w_{\sigma(k)}=0}=0$ for every $k=1, \ldots, N$.

Proof. We denote by $\operatorname{Prop}(N-\mu, N-v)$ a following proper holomorphic mapping:

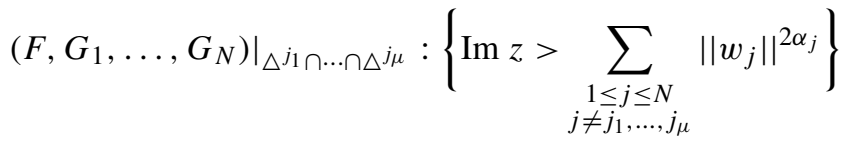

$$
\begin{aligned}
& \rightarrow\left\{\operatorname{Im} z>\sum_{\substack{1 \leq k \leq N \\
k \neq k_{1}, \ldots, k_{v}}}\left\|w_{k}\right\|^{2 \beta_{k}}\right\} .
\end{aligned}
$$


By Lemma 2.2, if we have Prop $(N-\mu, N-v)$, then, for any $k\left(1 \leq k \leq N, k \neq k_{1}, \ldots, k_{v}\right)$, there exists $j\left(1 \leq j \leq N, j \neq j_{1}, \ldots, j_{\mu}\right)$ such that

$$
\left.\left(F, G_{1}, \ldots, G_{N}\right)\right|_{\triangle^{j_{1} \cap \cdots \cap \triangle^{j_{\mu}}}}\left(\triangle^{j}\right) \subset \widetilde{\triangle}^{k} .
$$

We denote by $J(N-\mu, N-v)$ this correspondence $k \mapsto j$. First of all, as proved in Lemma 2.2, for any $k$, there exists $j$ such that $(F, G)\left(\triangle^{j}\right) \subset \widetilde{\triangle}^{k}$. Thus we have $J(N, N)$. We shall assert that $J(N, N)$ is injective; and hence, it is a permutation of indices. To this end, suppose that it is not injective. Then there would exist $k_{1}$ and $k_{2}$ such that $J(N, N)\left(k_{1}\right)=$ $J(N, N)\left(k_{2}\right)\left(=j_{1}\right)$ and $k_{1} \neq k_{2}$. Then there exists a proper holomorphic mapping $\operatorname{Prop}(N-$ $1, N-2)$ :

$$
\left.\left(F, G_{1}, \ldots, G_{N}\right)\right|_{\triangle^{j_{1}}}:\left\{\operatorname{Im} z>\sum_{\substack{1 \leq j \leq N \\ j \neq j_{1}}}\left\|w_{j}\right\|^{2 \alpha_{j}}\right\} \rightarrow\left\{\operatorname{Im} z>\sum_{\substack{1 \leq k \leq N \\ k \neq k_{1}, k_{2}}}\left\|w_{k}\right\|^{2 \beta_{k}}\right\} .
$$

By applying the same argument to this mapping, there exists a correspondence $J(N-1, N-$ 2). If it is injective, we have $\operatorname{Prop}(N-2, N-3)$ and if it is not injective, we have $\operatorname{Prop}(N-$ $2, N-4)$. Repeat this process. Then finally we obtain $\operatorname{Prop}\left(N-N_{0}, 0\right)$ for some $N_{0}$, which is a contradiction; proving our assertion.

On the boundary of $\Omega(m ;(m) ;(\alpha))$, we employ the usual coordinates $(x, w) \in \mathbf{R} \times \mathbf{C}^{m}$, that is, we identify naturally $(x, w)$ with $\left(x+i\||| w \mid\|^{2 \alpha}, w\right)$ throughout this paper. Using this coordinate, we define $\mathrm{CR}$ vector fields $L_{j}^{\lambda}$ by

$$
L_{j}^{\lambda}=\frac{\partial}{\partial w_{j}^{\lambda}}+i \alpha_{j}\left\|w_{j}\right\|^{2\left(\alpha_{j}-1\right)} \bar{w}_{j}^{\lambda} \frac{\partial}{\partial x}, \quad j=1, \ldots, N, \quad \lambda=1, \ldots, m_{j} .
$$

Extend $F$ and $G$ holomorphically past the boundary and we use the same symbol $(F, G)$ for the extended mapping. Expand the mapping $(F, G)$ as

$$
\begin{aligned}
F & =\sum_{|p|+q \geq 0} a_{p, q}(w)^{p} z^{q}, \\
G_{j}^{\lambda} & =\sum_{|p|+q \geq 0} b_{j ; p, q}^{\lambda}(w)^{p} z^{q}, \quad j=1, \ldots, N, \lambda=1, \ldots, n_{j} .
\end{aligned}
$$

We obtain the CR mapping by restricting them to the boundary and their expansions are the following

$$
\begin{aligned}
F & =\sum_{|p|+q \geq 0} a_{p, q}(w)^{p}\left(x+i\left|\|w \mid\|^{2 \alpha}\right)^{q},\right. \\
G_{j}^{\lambda} & =\sum_{|p|+q \geq 0} b_{j ; p, q}^{\lambda}(w)^{p}\left(x+\left.i|\| w|\right|^{2 \alpha}\right)^{q}, \quad j=1, \ldots, N, \lambda=1, \ldots, n_{j} .
\end{aligned}
$$

We use the notation $(F, G)$ as the extended holomorphic mapping and also as the CR mapping induced on the boundary. 
LEMMA 2.4. We have an expansion

$$
F(x, w)=\sum_{q \geq 0} a_{q}\left(x+i\left|\|w \mid\|^{2 \alpha}\right)^{q}, \quad a_{q} \in \mathbf{R} .\right.
$$

Proof. By making use of Lemma 2.3, we have $(F, G)\left(\triangle^{1} \cap \cdots \cap \triangle^{N}\right)=\widetilde{\triangle}^{1} \cap \cdots \cap \widetilde{\triangle}^{N}$. This means that $G(x, 0)=0$. We substitute (31) and (32) into $\operatorname{Im} F=\||| G \mid\|^{2 \beta}$ and apply CR vector fields to it. Then $L_{j_{k}}^{\lambda_{k}} \ldots L_{j_{1}}^{\lambda_{1}} \mid\|G\| \|^{2 \beta}$ is a summation of the terms which contain $L_{i_{k}}^{\mu_{k}} \ldots L_{i_{\ell+1}}^{\mu_{\ell+1}} G_{j}^{\lambda} \cdot L_{i_{\ell}}^{\mu_{\ell}} \ldots L_{i_{1}}^{\mu_{1}} \bar{G}_{j}^{\lambda}$ for suitable indices $j, \lambda, \ell$, and $i_{1}, \ldots, i_{k}, \mu_{1}, \ldots, \mu_{k}$. If $k=\ell$ (resp. $\ell=0$ ), then $L_{i_{k}}^{\mu_{k}} \ldots L_{i_{\ell+1}}^{\mu_{\ell+1}} G_{j}^{\lambda}$ (resp. $L_{i_{\ell}}^{\mu_{\ell}} \ldots L_{i_{1}}^{\mu_{1}} \bar{G}_{j}^{\lambda}$ ) is understood to be $G_{j}^{\lambda}$ (resp. $\bar{G}_{j}^{\lambda}$ ). The terms with $\ell=0$ vanish at $(x, 0)$. The terms with $\ell \neq 0$ contain $L_{i_{\ell}}^{\mu_{\ell}} \ldots L_{i_{1}}^{\mu_{1}} \bar{G}_{j}^{\lambda}$ and we show that they vanish at $(x, 0)$ as follows. Since we have

$$
L_{i_{\ell}}^{\mu_{\ell}} \ldots L_{i_{1}}^{\mu_{1}}=\frac{\partial^{\ell}}{\partial w_{i_{\ell}}^{\mu_{\ell}} \ldots \partial w_{i_{1}}^{\mu_{1}}}+\bar{w}_{i_{1}}^{\mu_{1}} P_{1}+\cdots+\bar{w}_{i_{\ell}}^{\mu_{\ell}} P_{\ell},
$$

where $P_{1}, \ldots, P_{\ell}$ are differential operators with order less than or equal to $\ell$, we calculate $L_{i_{\ell}}^{\mu_{\ell}} \ldots L_{i_{1}}^{\mu_{1}} \bar{G}_{j}^{\lambda}(x, 0)$ as follows.

$$
\begin{aligned}
\left(L_{i_{\ell}}^{\mu_{\ell}}\right. & \left.\ldots L_{i_{1}}^{\mu_{1}} \bar{G}_{j}^{\lambda}\right)(x, 0) \\
& =\left(\frac{\partial^{\ell}}{\partial w_{i_{\ell}}^{\mu_{\ell}} \ldots \partial w_{i_{1}}^{\mu_{1}}} \bar{G}_{j}^{\lambda}\right)(x, 0) \\
& =\left(\sum_{|p|+q \geq 0} \overline{b_{j ; p, q}^{\lambda}(w)^{p}} \frac{\partial^{\ell}}{\partial w_{i_{\ell}}^{\mu_{\ell}} \ldots \partial w_{i_{1}}^{\mu_{1}}}\left(x-\left.i|||w|\right|^{2 \alpha}\right)^{q}\right)(x, 0) \\
& =\sum_{q \geq 0} \bar{b}_{j ; 0, q}^{\lambda}\left(\frac{\partial^{\ell}}{\partial w_{i_{\ell}}^{\mu_{\ell}} \ldots \partial w_{i_{1}}^{\mu_{1}}}\left(x-\left.i|||w|\right|^{2 \alpha}\right)^{q}\right)(x, 0) \\
& =0 .
\end{aligned}
$$

Now we have $\left(L_{j_{k}}^{\lambda_{k}} \ldots L_{j_{1}}^{\lambda_{1}} \mid\|G\|^{2 \beta}\right)(x, 0)=0$. Therefore we see that

$$
\begin{aligned}
0 & =\left(L_{j_{k}}^{\lambda_{k}} \ldots L_{j_{1}}^{\lambda_{1}} \|\left.|G|\right|^{2 \beta}\right)(x, 0) \\
& =\left(L_{j_{k}}^{\lambda_{k}} \ldots L_{j_{1}}^{\lambda_{1}} \operatorname{Im} F\right)(x, 0) \\
& =\frac{1}{2 i}\left(L_{j_{k}}^{\lambda_{k}} \ldots L_{j_{1}}^{\lambda_{1}} F\right)(x, 0) . \\
& =\frac{1}{2 i} \frac{\partial^{k} F}{\partial w_{j_{k}}^{\lambda_{k}} \ldots \partial w_{j_{1}}^{\lambda_{1}}}(x, 0) .
\end{aligned}
$$


Here, in order to derive the third equality, we have used the same calculation as in (35). This implies that $a_{p, q}=0$ for $|p|>0$. We replace $a_{0, q}$ by $a_{q}$. The fact that $F(x, 0)$ is real implies that $a_{q} \in \mathbf{R}$.

REMARK 2. If there are some square norm terms in $\left.\||w|\|\right|^{2 \alpha}$, say $\alpha_{1}=1$ for instance, then this proof does not work. If $\alpha_{1}=1$, then Lemma 2.3 holds for $\sigma(i)=2, \ldots, N$, not $\sigma(i)=1, \ldots, N$. Therefore we do not have $G(x, 0)=0$.

\section{Relation between the exponents $\alpha_{1}$ and $\beta_{1}$}

From here to the next section, we assume that $N=1$, namely, the number of the block of $w$ is one.

LEMMA 3.1. If $\alpha_{1}>\beta_{1}$, then there exists $M \in \mathbf{N}$ such that $\alpha_{1}=M \beta_{1}$.

Proof. Suppose fails. Then there exists $M_{0} \in \mathbf{N}$ such that $M_{0} \beta_{1}<\alpha_{1}<\left(M_{0}+1\right) \beta_{1}$. First we claim that the following equality holds

$$
\left(L_{1}^{\lambda_{k}} \cdots L_{1}^{\lambda_{1}}\right) G_{1}^{1}(x, 0)=\cdots=\left(L_{1}^{\lambda_{k}} \cdots L_{1}^{\lambda_{1}}\right) G_{1}^{n_{1}}(x, 0)=0
$$

for any $k=1, \ldots, M_{0}$ and $\lambda_{1}, \ldots, \lambda_{k} \in\left\{1, \ldots, m_{1}\right\}$. This is proved by induction on $k$ as follows. From Lemma 2.4, we obtain the following equation:

$$
\begin{aligned}
\left(L_{1}^{\lambda_{k}}\right)^{\beta_{1}} \cdots\left(L_{1}^{\lambda_{1}}\right)^{\beta_{1}} F= & \sum_{\ell=1}^{k \beta_{1}} \sum_{q \geq \ell} \text { (non-zero constant) }\left\|w_{1}\right\|^{2\left(\ell \alpha_{1}-k \beta_{1}\right)} \\
& \times\left(\bar{w}_{1}^{\lambda_{1}}\right)^{\beta_{1}} \cdots\left(\bar{w}_{1}^{\lambda_{k}}\right)^{\beta_{1}}\left(x+i\left\|w_{1}\right\|^{2 \alpha_{1}}\right)^{q-\ell} .
\end{aligned}
$$

The lowest degree of $\bar{w}_{1}$ in the equation (38) is $\ell \alpha_{1}$, which is greater than $M_{0} \beta_{1}$. Therefore by applying $\left(\bar{L}_{1}^{\lambda_{k}}\right)^{\beta_{1}} \ldots\left(\bar{L}_{1}^{\lambda_{1}}\right)^{\beta_{1}}$ to this equation and by evaluating at $(x, 0)$, we obtain

$$
\left(\bar{L}_{1}^{\lambda_{k}}\right)^{\beta_{1}} \cdots\left(\bar{L}_{1}^{\lambda_{1}}\right)^{\beta_{1}}\left(L_{1}^{\lambda_{k}}\right)^{\beta_{1}} \cdots\left(L_{1}^{\lambda_{1}}\right)^{\beta_{1}} F(x, 0)=0
$$

for $k=1, \ldots, M_{0}$. Applying $\left(\bar{L}_{1}^{\lambda_{1}}\right)^{\beta_{1}}\left(L_{1}^{\lambda_{1}}\right)^{\beta_{1}}$ to the equation $\operatorname{Im} F=\left\|G_{1}\right\|^{2 \beta_{1}}$, we get the relation

$$
\begin{aligned}
& \left(\bar{L}_{1}^{\lambda_{1}}\right)^{\beta_{1}}\left(L_{1}^{\lambda_{1}}\right)^{\beta_{1}} F \\
& \quad=\text { (positive constant) }\left(\sum_{\lambda=1}^{n_{1}}\left|L_{1}^{\lambda_{1}} G_{1}^{\lambda}\right|^{2}\right)^{\beta_{1}}+\left\{\text { the terms containing }\left\|G_{1}\right\|\right\} .
\end{aligned}
$$

By evaluating at $(x, 0)$, this together with (39) and $G_{1}(x, 0)=0$, we obtain (37) for $k=1$. Now we assume that (37) holds for $k=k_{0}$. We apply CR vectors and their complex conjugate $\left(\bar{L}_{1}^{\lambda_{k_{0}+1}}\right)^{\beta_{1}} \cdots\left(\bar{L}_{1}^{\lambda_{1}}\right)^{\beta_{1}}\left(L_{1}^{\lambda_{k_{0}+1}}\right)^{\beta_{1}} \cdots\left(L_{1}^{\lambda_{1}}\right)^{\beta_{1}}$ to $\operatorname{Im} F=\left\|G_{1}\right\|^{2 \beta_{1}}$ and evaluate at $(x, 0)$. Then we get

$$
0=\left(\bar{L}_{1}^{\lambda_{k_{0}+1}}\right)^{\beta_{1}} \cdots\left(\bar{L}_{1}^{\lambda_{1}}\right)^{\beta_{1}}\left(L_{1}^{\lambda_{k_{0}+1}}\right)^{\beta_{1}} \cdots\left(L_{1}^{\lambda_{1}}\right)^{\beta_{1}} F(x, 0)
$$




$$
=(\text { positive constant })\left(\sum_{\lambda=1}^{n_{1}}\left|L_{1}^{\lambda_{k_{0}+1}} \cdots L_{1}^{\lambda_{1}} G_{1}^{\lambda}(x, 0)\right|^{2}\right)^{\beta_{1}},
$$

which implies (37) for $k=k_{0}+1$. This process continues up to $k=M_{0}$. This proves (37). By (37), $G_{1}^{\lambda}$ can be expanded as

$$
G_{1}^{\lambda}=\sum_{\substack{|p| \geq M_{0}+1 \\ q \geq 0}} b_{1 ; p, q}^{\lambda}\left(w_{1}\right)^{p}\left(x+i\left\|w_{1}\right\|^{2 \alpha_{1}}\right)^{q} .
$$

Substitute (33) and (42) into $\operatorname{Im} F=\left\|G_{1}\right\|^{2 \beta_{1}}$ and pick up the $\left\|w_{1}\right\|^{2 \alpha_{1}}$ terms. Then we obtain the following equation

$$
\sum_{q \geq 1} a_{q} q x^{q-1}\left\|w_{1}\right\|^{2 \alpha_{1}}=0
$$

which implies $F=a_{0}$. This is a contradiction.

Lemma 3.2. Assume that $\alpha_{1} \leq \beta_{1}$. Then we have $\alpha_{1}=\beta_{1}$.

Proof. Assume that the equality does not hold. Substitute (32) and (33) into $\operatorname{Im} F=$ $\left\|G_{1}\right\|^{2 \beta_{1}}$ and pick up the terms without $w_{1}$ or $\bar{w}_{1}$. Then we have

$$
0=\left|\sum_{q \geq 0} b_{1 ; 0, q^{1}}^{1} x^{q}\right|^{2}+\cdots+\left|\sum_{q \geq 0} b_{1 ; 0, q}^{n_{1}} x^{q}\right|^{2},
$$

which implies $b_{1 ; 0, q}^{\lambda}=0$ for $\lambda=1, \ldots, n_{1}$ and $q \geq 0$. Plug these into $\operatorname{Im} F=\left\|G_{1}\right\|^{2 \beta_{1}}$ and pick up the terms of total degree $2 \alpha_{1}$ in $w_{1}$ and $\bar{w}_{1}$. Noting $\alpha_{1}<\beta_{1}$, we have $a_{q}=0$ for $q \geq 1$. This implies $F=a_{0}$; a contradiction. Now we have $\alpha_{1}=\beta_{1}$.

From Lemmas 3.1 and 3.2, in any case, there exists $M \in \mathbf{N}$ such that $\alpha_{1}=M \beta_{1}$.

\section{Expansions of $F$ and $G$ (one block case)}

In this section, we still continue to assume $N=1$. We shall obtain expansions of $F$ and $G_{1}$ for $\alpha_{1}=M \beta_{1}$ and conclude that $M=1$ if $3<n_{1}<2 m_{1}-1$.

Substituting (32) and (33) into $\operatorname{Im} F=\left\|G_{1}\right\|^{2 \beta_{1}}$ and comparing the terms of total degree $0,2 \beta_{1}, 4 \beta_{1}, \ldots, 2(M-1) \beta_{1}$ of $w_{1}$ and $\bar{w}_{1}$ in the both sides, we obtain the expansion

$$
G_{1}^{\lambda}=\sum_{\substack{|p| \geq M \\ q \geq 0}} b_{1 ; p, q}^{\lambda}\left(w_{1}\right)^{p}\left(x+i\left\|w_{1}\right\|^{2 M \beta_{1}}\right)^{q} .
$$

LEMMA 4.1. Assume that $a_{1}=a_{2}=\cdots=a_{2(k-1) \beta_{1}+1}=0$ in (33). Then we have expansions

$$
F=a_{0}+\sum_{q \geq 2 k \beta_{1}+1} a_{q}\left(x+i\left\|w_{1}\right\|^{2 M \beta_{1}}\right)^{q},
$$




$$
\begin{aligned}
G_{1}^{\lambda}= & \sum_{\substack{|p|=M \\
q \geq k}} b_{1 ; p, q}^{\lambda}\left(w_{1}\right)^{p}\left(x+i\left\|w_{1}\right\|^{2 M \beta_{1}}\right)^{q} \\
& +\sum_{\substack{|p| \geq M+1 \\
q \geq 0}} b_{1 ; p, q}^{\lambda}\left(w_{1}\right)^{p}\left(x+i\left\|w_{1}\right\|^{2 M \beta_{1}}\right)^{q} .
\end{aligned}
$$

Proof. We use the expansions (33) and (45). First we assume that $a_{1}=0$. Pick up the terms of total degree $2 M \beta_{1}$ of $w_{1}$ and $\bar{w}_{1}$ and degree zero in $x$ from $\operatorname{Im} F=\left\|G_{1}\right\|^{2 \beta_{1}}$. Then we have $b_{1 ; p, 0}^{\lambda}=0$ for $|p|=M$. Substituting them into $\operatorname{Im} F=\left\|G_{1}\right\|^{2 \beta_{1}}$ and comparing the terms of total degree $2 M \beta_{1}$ of $w_{1}$ and $\bar{w}_{1}$, we obtain $a_{q}=0$ for $q=2, \ldots, 2 \beta_{1}$. This proves the case of $k=1$. Suppose that lemma holds for $k=k_{0}$. Assume that $a_{1}=a_{2}=\cdots=$ $a_{2 k_{0} \beta_{1}+1}=0$. We substitute (46) and (47) with $k=k_{0}$ into $\operatorname{Im} F=\left\|G_{1}\right\|^{2 \beta_{1}}$ and pick up the terms of $w_{1}$ and $\bar{w}_{1}$ with total degree $2 M \beta_{1}$. Then we obtain the equation

$$
\begin{aligned}
& \left.\sum_{q \geq 2 k_{0} \beta_{1}+2} a_{q} q x^{q-1}|| w_{1}\right|^{2 M \beta_{1}} \\
& \quad=\left(\left|\sum_{\substack{|p|=M \\
q \geq k_{0}}} b_{1 ; p, q}^{1}\left(w_{1}\right)^{p} x^{q}\right|^{2}+\cdots+\left|\sum_{\substack{|p|=M \\
q \geq k_{0}}} b_{1 ; p, q}^{n_{1}}\left(w_{1}\right)^{p} x^{q}\right|^{2}\right)^{\beta_{1}},
\end{aligned}
$$

which implies that $b_{1 ; p, k_{0}}^{\lambda}=0$ for $|p|=M$ and $\lambda=1, \ldots, n_{1}$. Substituting them into $\operatorname{Im} F=\left\|G_{1}\right\|^{2 \beta_{1}}$ and comparing the terms of $w_{1}$ and $\bar{w}_{1}$ with total degree $2 M \beta_{1}$, we obtain $a_{q}=0$ for $q=2 k_{0} \beta_{1}+2, \ldots, 2\left(k_{0}+1\right) \beta_{1}$. This completes the proof.

From this lemma, we assume that there exists $k$ such that $a_{1}=a_{2}=\cdots=$ $a_{2(k-1) \beta_{1}+1}=0$ and $a_{2 k \beta_{1}+1} \neq 0$. Then we have expansions (46) and (47). The coefficients of $\left|w_{1}^{1}\right|^{2 M \beta_{1}} x^{2 k \beta_{1}}, \ldots,\left|w_{1}^{m_{1}}\right|^{2 M \beta_{1}} x^{2 k \beta_{1}}$ and $\left(w_{1}^{i} \bar{w}_{1}^{j}\right)^{M \beta_{1}} x^{2 k \beta_{1}}$ for $i \neq j$ in $\operatorname{Im} F=\left\|G_{1}\right\|^{2 \beta_{1}}$ satisfy

$$
\begin{gathered}
\left(2 k \beta_{1}+1\right) a_{2 k \beta_{1}+1}=\left(\left|b_{1 ;(M, 0, \ldots, 0), k}^{1}\right|^{2}+\cdots+\left|b_{1 ;(M, 0, \ldots, 0), k}^{n_{1}}\right|^{2}\right)^{\beta_{1}} \\
\vdots \\
=\left(\left|b_{1 ;(0, \ldots, 0, M), k}^{1}\right|^{2}+\cdots+\left|b_{1 ;(0, \ldots, 0, M), k}^{n_{1}}\right|^{2}\right)^{\beta_{1}}
\end{gathered}
$$

and

$$
0=b_{1 ;(0, \ldots, M, \ldots, 0), k}^{1} \bar{b}_{1 ;(0, \ldots, M, \ldots, 0), k}^{1}+\cdots+b_{1 ;(0, \ldots, M, \ldots, 0), k}^{n_{1}} \bar{b}_{1 ;(0, \ldots, M, \ldots, 0), k}^{n_{1}} .
$$

Here, in the lower indices of $b_{1 ;(0, \ldots, M, \ldots, 0), k}^{\lambda}$ (resp. $\left.\bar{b}_{1 ;(0, \ldots, M, \ldots, 0), k}^{\lambda}\right)$ in (50), $M$ appears in the $i$-th (resp. $j$-th) component and the other components are zero. Therefore the vectors

$$
U_{j}=\left(\frac{b_{1 ;(0, \ldots, M, \ldots, 0), k}^{1}}{\left\{\left(2 k \beta_{1}+1\right) a_{2 k \beta_{1}+1}\right\}^{1 / 2 \beta_{1}}}, \ldots, \frac{b_{1 ;(0, \ldots, M, \ldots, 0), k}^{n_{1}}}{\left\{\left(2 k \beta_{1}+1\right) a_{2 k \beta_{1}+1}\right\}^{1 / 2 \beta_{1}}}\right),
$$


for $j=1, \ldots, m_{1}$, satisfy $<U_{i}, U_{j}>=\delta_{i}^{j}$. Here, in the lower indices $(0, \ldots, M, \ldots, 0)$ appearing in $U_{j}, M$ appears in the $j$-th component. Therefore we can find vectors $U_{m_{1}+1}, \ldots, U_{n_{1}}$ such that the matrix

$$
U=\left(\begin{array}{c}
U_{1} \\
\vdots \\
U_{n_{1}}
\end{array}\right)
$$

is unitary.

Define $\left(F^{*}, G_{1}^{1 *}, \ldots, G_{1}^{n_{1 *}}\right)$ as

$$
\begin{aligned}
& \left(F^{*}, G_{1}^{1 *}, \ldots, G_{1}^{n_{1} *}\right) \\
& \quad=\left(F, G_{1}^{1}, \ldots, G_{1}^{n_{1}}\right)\left(\begin{array}{cc}
\frac{1}{\left(2 k \beta_{1}+1\right) a_{2 k \beta_{1}+1}} & 0 \\
0 & \frac{1}{\left\{\left(2 k \beta_{1}+1\right) a_{2 k \beta_{1}+1}\right\}^{1 / 2 \beta_{1}}} \bar{U}^{t}
\end{array}\right) .
\end{aligned}
$$

Then the expansions of $F^{*}, G_{1}^{1 *}, \ldots, G_{1}^{n_{1} *}$ are of the forms

$$
\begin{aligned}
& F^{*}=\frac{a_{0}}{\left(2 k \beta_{1}+1\right) a_{2 k \beta_{1}+1}}+\frac{1}{2 k \beta_{1}+1}\left(x+i\left\|w_{1}\right\|^{2 M \beta_{1}}\right)^{2 k \beta_{1}+1} \\
& +\sum_{q \geq 2 k \beta_{1}+2} a_{q}^{*}\left(x+i\left\|w_{1}\right\|^{2 M \beta_{1}}\right)^{q}, \\
& G_{1}^{\lambda *}=\left(w_{1}^{\lambda}\right)^{M}\left(x+i\left\|w_{1}\right\|^{2 M \beta_{1}}\right)^{k}+\sum_{\substack{|p|=M \\
\text { one of } p_{j}=M \\
q \geq k+1}} b_{1 ; p, q}^{\lambda *}\left(w_{1}\right)^{p}\left(x+i\left\|w_{1}\right\|^{2 M \beta_{1}}\right)^{q} \\
& +\sum_{\substack{|p|=M \\
p_{1}, \ldots, p_{m_{1}} \neq M \\
q \geq k}} b_{1 ; p, q}^{\lambda *}\left(w_{1}\right)^{p}\left(x+i\left\|w_{1}\right\|^{2 M \beta_{1}}\right)^{q} \\
& +\sum_{\substack{|p| \geq M+1 \\
q \geq 0}} b_{1 ; p, q}^{\lambda *}\left(w_{1}\right)^{p}\left(x+i\left\|w_{1}\right\|^{2 M \beta_{1}}\right)^{q}, \quad \lambda=1, \ldots, m_{1}, \\
& G_{1}^{\lambda *}=\sum_{\substack{|p|=M \\
\text { one of } p_{j}=M \\
q \geq k+1}} b_{1 ; p, q}^{\lambda *}\left(w_{1}\right)^{p}\left(x+i|| w_{1}||^{2 M \beta_{1}}\right)^{q} \\
& +\sum_{\substack{|p|=M \\
p_{1}, \ldots, p_{m_{1}} \neq M \\
q \geq k}} b_{1 ; p, q}^{\lambda *}\left(w_{1}\right)^{p}\left(x+i\left\|w_{1}\right\|^{2 M \beta_{1}}\right)^{q}
\end{aligned}
$$




$$
+\sum_{\substack{|p| \geq M+1 \\ q \geq 0}} b_{1 ; p, q}^{\lambda *}\left(w_{1}\right)^{p}\left(x+i\left\|w_{1}\right\|^{2 M \beta_{1}}\right)^{q}, \quad \lambda=m_{1}+1, \ldots, n_{1} .
$$

If $M=1$, we understand that the third (resp. second) term on the right hand side in (55) (resp. in (56)) is vacuum. Next steps are to simplify the coefficients of $F^{*}, G_{1}^{1 *}, \ldots, G_{1}^{n_{1 *}}$ and to determine $M$. In the following, we drop $*$ in (54), (55) and (56).

LEMMA 4.2. We have $k=0, \alpha_{1}=\beta_{1}$. The mapping $\left(F, G_{1}\right)$ is equivalent to $\left(z, w_{1}, 0\right)$.

Proof. Substitute the expansions (54), (55) and (56) into $\operatorname{Im} F=\left\|G_{1}\right\|^{2 \beta_{1}}$ and put $x=0$. The minimal value of the total degree of $w_{1}$ and $\bar{w}_{1}$ on the left hand side is $2 M \beta_{1}\left(2 k \beta_{1}+1\right)$, which comes from

$$
\operatorname{Im} \frac{1}{2 k \beta_{1}+1}\left(x+i\left\|w_{1}\right\|^{2 M \beta_{1}}\right)^{2 k \beta_{1}+1} .
$$

Therefore the terms of total degrees $2 \beta_{1}(M+1), 2 \beta_{1}(M+2), \ldots, 2 \beta_{1}\left(M+2 M \beta_{1} k-1\right)$ of $w_{1}$ and $\bar{w}_{1}$ on the right hand side, which come from

$$
\sum_{\substack{|p| \geq M+1 \\ q \geq 0}} b_{1 ; p, q}^{\lambda}\left(w_{1}\right)^{p}\left(i\left\|w_{1}\right\|^{2 M \beta_{1}}\right)^{q},
$$

are zero. Hence, the ranges of the indices $(p, q)$ in the summation (58) become

$$
\begin{aligned}
|p| & \geq M+2 M \beta_{1} k, \quad q \geq 0, \\
|p| & =M+2 M \beta_{1}(k-1), \ldots, M+2 M \beta_{1} k-1, \quad q \geq 1, \\
|p| & =M+2 M \beta_{1}(k-2), \ldots, M+2 M \beta_{1}(k-1)-1, \quad q \geq 2, \\
& \vdots \\
|p| & =M+2 M \beta_{1} \cdot 2, \ldots, M+2 M \beta_{1} \cdot 3-1, \quad q \geq k-2, \\
|p| & =M+2 M \beta_{1}, \ldots, M+2 M \beta_{1} \cdot 2-1, \quad q \geq k-1 \\
|p| & =M+1, \ldots, M+2 M \beta_{1}-1, \quad q \geq k .
\end{aligned}
$$

Assume that $k \neq 0$. Plug these indices into (58) and restrict $\operatorname{Im} F=\left\|G_{1}\right\|^{2 \beta_{1}}$ to $x=0$. Collect the terms of total degree $2 M \beta_{1}\left(2 k \beta_{1}+1\right)$ of $w_{1}$ and $\bar{w}_{1}$ in the restricted equation. Among these terms, we compare the degree of $\left\|w_{1}\right\|$. Since the degree of $\left\|w_{1}\right\|$ on the left hand side is $2 M \beta_{1}\left(2 k \beta_{1}+1\right)$, the coefficients of $\left\|w_{1}\right\|^{0},\left\|w_{1}\right\|^{4 M \beta_{1}^{2}}, \ldots,\left\|w_{1}\right\|^{4 M \beta_{1}^{2}(k-1)}$ are zero. Therefore in the summation (58) with indices from (59) to (64), the terms with indices

$$
\begin{aligned}
(|p|, q)= & \left(M+2 M \beta_{1} k, 0\right),\left(M+2 M \beta_{1}(k-1), 1\right),\left(M+2 M \beta_{1}(k-2), 2\right), \ldots, \\
& \left(M+2 M \beta_{1} \cdot 2, k-2\right),\left(M+2 M \beta_{1}, k-1\right)
\end{aligned}
$$


do not appear. Consequently, the indices in (58) become

$$
\begin{aligned}
& |p| \geq M+2 M \beta_{1} k+1, \quad q \geq 0, \\
& |p|=M+2 M \beta_{1}(k-1)+1, \ldots, M+2 M \beta_{1} k, \quad q \geq 1, \\
& |p|=M+2 M \beta_{1}(k-2)+1, \ldots, M+2 M \beta_{1}(k-1), \quad q \geq 2, \\
& \quad \vdots \\
& |p|=M+2 M \beta_{1} \cdot 2+1, \ldots, M+2 M \beta_{1} \cdot 3, \quad q \geq k-2, \\
& |p|=M+2 M \beta_{1}+1, \ldots, M+2 M \beta_{1} \cdot 2, \quad q \geq k-1, \\
& |p|=M+1, \ldots, M+2 M \beta_{1}, \quad q \geq k .
\end{aligned}
$$

Plug these into (58) and pick up the terms of total degree $2 M \beta_{1}\left(2 k \beta_{1}+1\right)$ of $w_{1}$ and $\bar{w}_{1}$ from the equation $\operatorname{Im} F=\left\|G_{1}\right\|^{2 \beta_{1}}$ which is restricted to $x=0$. Divide them by $\left\|w_{1}\right\|^{4 M \beta_{1}^{2} k}$ and take $\beta_{1}$-th root. Then we get

$$
\begin{aligned}
& \operatorname{Im} \frac{1}{\left(2 k \beta_{1}+1\right)^{1 / \beta_{1}}} \|\left. w_{1}\right|^{2 M_{i}\left(2 \beta_{1} k+1\right) / \beta_{1}} \\
& =\left|\left(w_{1}^{1}\right)^{M}+\sum_{\substack{|p|=M \\
p_{1}, \ldots, p_{m_{1}} \neq M}} b_{1 ; p, k}^{1}\left(w_{1}\right)^{p}\right|^{2}+\ldots \\
& \quad+\left|\left(w_{1}^{m_{1}}\right)^{M}+\sum_{\substack{|p|=M \\
p_{1}, \ldots, p_{m_{1}} \neq M}} b_{1 ; p, k}^{m_{1}}\left(w_{1}\right)^{p}\right|^{2} \\
& \quad+\left|\sum_{\substack{|p|=M \\
p_{1}, \ldots, p_{m_{1}} \neq M}} b_{1 ; p, k}^{m_{1}+1}\left(w_{1}\right)^{p}\right|^{2}+\cdots+\left.\sum_{\substack{|p|=M \\
p_{1}, \ldots, p_{m_{1}} \neq M}} b_{1 ; p, k}^{n_{1}}\left(w_{1}\right)^{p}\right|^{2} .
\end{aligned}
$$

Comparing the coefficients of $\left(w_{1}^{1}\right)^{M}, \ldots,\left(w_{1}^{m_{1}}\right)^{M}$, we conclude that the summations

$$
\sum_{\substack{|p|=M \\ p_{1}, \ldots, p_{m_{1}} \neq M}} b_{1 ; p, k}^{1}\left(w_{1}\right)^{p}, \ldots, \sum_{\substack{|p|=M \\ p_{1}, \ldots, p_{m_{1}} \neq M}} b_{1 ; p, k}^{m_{1}}\left(w_{1}\right)^{p}
$$

do not appear and

$$
\frac{1}{\left(2 k \beta_{1}+1\right)^{1 / \beta_{1}}}=1,
$$


namely, we have $k=0$. This means that the mapping

$$
\begin{gathered}
\Phi_{M}\left(w_{1}\right)=\left(\left(w_{1}^{1}\right)^{M}, \ldots,\left(w_{1}^{m_{1}}\right)^{M}, \sum_{\substack{|p|=M \\
p_{1}, \ldots, p_{m_{1}} \neq M}} b_{1 ; p, 0}^{m_{1}+1}\left(w_{1}\right)^{p}, \ldots,\right. \\
\left.\sum_{\substack{|p|=M \\
p_{1}, \ldots, p_{m_{1}} \neq M}} b_{1 ; p, 0}^{n_{1}}\left(w_{1}\right)^{p}\right)
\end{gathered}
$$

is a proper holomorphic mapping $\Phi_{M}: B^{m_{1}} \rightarrow B^{n_{1}}$ of homogeneous degree $M$. By the gap theorem for balls with $3<n_{j}<2 m_{j}-1$, we can find $\Phi_{m_{1}} \in \operatorname{Aut}\left(B^{m_{1}}\right)$ and $\Phi_{n_{1}} \in \operatorname{Aut}\left(B^{n_{1}}\right)$ such that $\Phi_{n_{1}} \circ\left(w_{1}, 0\right) \circ \Phi_{m_{1}}\left(w_{1}\right)=\Phi_{M}\left(w_{1}\right)$. Therefore the proper holomorphic mapping $\Phi_{M}: B^{m_{1}} \rightarrow B^{n_{1}}$ is injective and its Jacobian matrix, denoted by $M_{\Phi_{M}}\left(w_{1}\right)$, has maximal rank at every point $w_{1} \in B^{m_{1}}$, especially, we have rank $M_{\Phi_{M}}(0)=m_{1}$. By (75), the Jacobian matrix $M_{\Phi_{M}}\left(w_{1}\right)$ can be written in the form

$$
M_{\Phi_{M}}\left(w_{1}\right)=\left(\begin{array}{cccc}
M\left(w_{1}^{1}\right)^{M-1} & 0 & \ldots & 0 \\
0 & M\left(w_{1}^{2}\right)^{M-1} & \ldots & 0 \\
\vdots & \vdots & \ddots & \vdots \\
0 & 0 & \ldots & M\left(w_{1}^{m_{1}}\right)^{M-1} \\
* & * & \ldots & * \\
\vdots & \vdots & \ddots & \vdots
\end{array}\right) .
$$

Note that the components from the $\left(m_{1}+1\right)$-st row to the $n_{1}$-th row are not necessarily zero. Assume that $M>1$. Then we have the following inequalities:

$$
\begin{aligned}
\operatorname{rank} M_{\Phi_{M}}(0) & =\operatorname{rank}\left(\begin{array}{ccc}
0 & \ldots & 0 \\
\vdots & \ddots & \vdots \\
0 & \ldots & 0 \\
* & \ldots & * \\
\vdots & \ddots & \vdots
\end{array}\right) \\
& \leq n_{1}-m_{1}<\left(2 m_{1}-1\right)-m_{1} \\
& =m_{1}-1 .
\end{aligned}
$$

This is a contradiction. Therefore we have $M=1$, which means that $\alpha_{1}=\beta_{1}$. From now on, we use $\alpha_{1}$ instead of $\beta_{1}$. Next we shall show that $F$ is equivalent to $z$ and $G_{1}=\left(w_{1}, 0\right)$. Note that since $M=1$, the third (resp. second) term on the right hand side in (55) (resp. in (56)) is vacuum. Let

$$
g_{1 ; 1}^{\lambda}\left(x, w_{1}\right)=w_{1}^{\lambda}+\sum_{\substack{|p|=1 \\ q \geq 1}} b_{1 ; p, q}^{\lambda}\left(w_{1}\right)^{p} x^{q}, \quad \lambda=1, \ldots, m_{1},
$$




$$
\begin{aligned}
& g_{1 ; 1}^{\lambda}\left(x, w_{1}\right)=\sum_{\substack{|p|=1 \\
q \geq 1}} b_{1 ; p, q}^{\lambda}\left(w_{1}\right)^{p} x^{q}, \quad \lambda=m_{1}+1, \ldots, n_{1}, \\
& g_{1 ; \ell}^{\lambda}\left(x, w_{1}\right)=\sum_{\substack{|p|=\ell \\
q \geq 0}} b_{1 ; p, q}^{\lambda}\left(w_{1}\right)^{p} x^{q}, \quad \lambda=1, \ldots, n_{1}, \ell \geq 2,
\end{aligned}
$$

and

$$
G_{1}^{\lambda}\left(x, w_{1}\right)=\sum_{\ell \geq 1} g_{1 ; \ell}^{\lambda}\left(x+i\left\|w_{1}\right\|^{2 \alpha_{1}}, w_{1}\right), \quad \lambda=1, \ldots, n_{1} .
$$

Denote by $\tilde{g}_{1 ; \ell}^{\lambda}=g_{1 ; \ell}^{\lambda}\left(0, w_{1}\right)$. In our following argument, we pick up the terms with certain degree from $\operatorname{Im} F\left(x+i\left\|w_{1}\right\|^{2 \alpha_{1}}, w_{1}\right)=\left\|G_{1}\left(x+i\left\|w_{1}\right\|^{2 \alpha_{1}}, w_{1}\right)\right\|^{2 \alpha_{1}}$. We shall omit the variables if there is no confusion. The terms of total degree $2 \alpha_{1}$ of $w_{1}$ and $\bar{w}_{1}$ in $\operatorname{Im} F=$ $\left\|G_{1}\right\|^{2 \alpha_{1}}$ satisfy

$$
\left\|w_{1}\right\|^{2 \alpha_{1}}=\left(\left|g_{1 ; 1}^{1}\left(x, w_{1}\right)\right|^{2}+\cdots+\left|g_{1 ; 1}^{n_{1}}\left(x, w_{1}\right)\right|^{2}\right)^{\alpha_{1}} .
$$

Differentiate it by $x$ once and twice. Then we have

$$
\begin{aligned}
0= & \operatorname{Re}\left(g_{1 ; 1}^{1}\left(x, w_{1}\right) \sum_{\substack{|p|=1 \\
q \geq 1}} \overline{b_{1 ; p, q}^{1}\left(w_{1}\right)^{p}}\left(\begin{array}{l}
q \\
1
\end{array}\right) x^{q-1}+\cdots\right. \\
& \left.+g_{1 ; 1}^{n_{1}}\left(x, w_{1}\right) \sum_{\substack{|p|=1 \\
q \geq 1}} \overline{b_{1 ; p, q}^{n_{1}}\left(w_{1}\right)^{p}}\left(\begin{array}{l}
q \\
1
\end{array}\right) x^{q-1}\right)
\end{aligned}
$$

and

$$
\begin{aligned}
& 0=\left|\sum_{\substack{|p|=1 \\
q \geq 1}} b_{1 ; p, q}^{1}\left(w_{1}\right)^{p}\left(\begin{array}{l}
q \\
1
\end{array}\right) x^{q-1}\right|^{2}+\cdots+\left|\sum_{\substack{|p|=1 \\
q \geq 1}} b_{1 ; p, q}^{n_{1}}\left(w_{1}\right)^{p}\left(\begin{array}{c}
q \\
1
\end{array}\right) x^{q-1}\right|^{2} \\
& +2 \operatorname{Re}\left(g_{1 ; 1}^{1} \sum_{\substack{|p|=1 \\
q \geq 2}} \overline{b_{1 ; p, q}^{1}\left(w_{1}\right)^{p}}\left(\begin{array}{l}
q \\
2
\end{array}\right) x^{q-2}+\cdots\right. \\
& \left.+g_{1 ; 1}^{n_{1}} \sum_{\substack{|p|=1 \\
q \geq 2}} \overline{b_{1 ; p, q}^{n_{1}}\left(w_{1}\right)^{p}}\left(\begin{array}{l}
q \\
2
\end{array}\right) x^{q-2}\right)
\end{aligned}
$$

Since the total degrees of $w_{1}$ and $\bar{w}_{1}$ on the left hand side of $\operatorname{Im} F=\left\|G_{1}\right\|^{2 \alpha_{1}}$ are $2 \alpha_{1}, 6 \alpha_{1}, \ldots$, the terms of total degrees $2 \alpha_{1}+1, \ldots, 6 \alpha_{1}-1$ of them on the right hand side must be zero. First, we compare the total degrees of $w_{1}$ and $\bar{w}_{1}$ from $2 \alpha_{1}+1$ to $4 \alpha_{1}-1$. 
The total degree $2 \alpha_{1}+1$ terms satisfy

$$
\left(\left|g_{1 ; 1}^{1}\right|^{2}+\cdots+\left|g_{1 ; 1}^{n_{1}}\right|^{2}\right)^{\alpha_{1}-1} \operatorname{Re}\left(g_{1 ; 1}^{1} \bar{g}_{1 ; 2}^{1}+\cdots+g_{1 ; 1}^{n_{1}} \bar{g}_{1 ; 2}^{n_{1}}\right)=0,
$$

which means that $g_{1 ; 1}^{1} \bar{g}_{1 ; 2}^{1}+\cdots+g_{1 ; 1}^{n_{1}} \bar{g}_{1 ; 2}^{n_{1}}=0$. The total degree $2 \alpha_{1}+2$ terms satisfy

$$
\begin{aligned}
& \left(\left|g_{1 ; 1}^{1}\right|^{2}+\cdots+\left|g_{1 ; 1}^{n_{1}}\right|^{2}\right)^{\alpha_{1}-1} \\
& \quad \times\left\{\left|g_{1 ; 2}^{1}\right|^{2}+\cdots+\left|g_{1 ; 2}^{n_{1}}\right|^{2}+2 \operatorname{Re}\left(g_{1 ; 1}^{1} \bar{g}_{1 ; 3}^{1}+\cdots+g_{1 ; 1}^{n_{1}} \bar{g}_{1 ; 3}^{n_{1}}\right)\right\}=0,
\end{aligned}
$$

which implies $g_{1 ; 2}^{\lambda}=0, \lambda=1, \ldots, n_{1}$, and $g_{1 ; 1}^{1} \bar{g}_{1 ; 3}^{1}+\cdots+g_{1 ; 1}^{n_{1}} \bar{g}_{1 ; 3}^{n_{1}}=0$. Repeat this process up to total degree $4 \alpha_{1}-1$. By induction (we omit the proof), we obtain

$$
\begin{aligned}
& g_{1 ; \ell}^{1}=\cdots=g_{1 ; \ell}^{n_{1}}=0, \quad \ell=2, \ldots, \alpha_{1}, \\
& g_{1 ; 1}^{1} \bar{g}_{1 ; \ell}^{1}+\cdots+g_{1 ; 1}^{n_{1}} \bar{g}_{1 ; \ell}^{n_{1}}=0, \quad \ell=2,3, \ldots, 2 \alpha_{1} .
\end{aligned}
$$

The total degree $4 \alpha_{1}$ terms in $\operatorname{Im} F=\left\|G_{1}\right\|^{2 \alpha_{1}}$ satisfy

$$
\begin{aligned}
& \left(\left|g_{1 ; 1}^{1}\right|^{2}+\cdots+\left|g_{1 ; 1}^{n_{1}}\right|^{2}\right)^{\alpha_{1}-1} \\
& \times\left\{\left|g_{1 ; \alpha_{1}+1}^{1}\right|^{2}+\cdots+\left|g_{1 ; \alpha_{1}+1}^{n_{1}}\right|^{2}+2 \operatorname{Re}\left(g_{1 ; 1}^{1} \bar{g}_{1 ; 2 \alpha_{1}+1}^{1}+\cdots+g_{1 ; 1}^{n_{1}} \bar{g}_{1 ; 2 \alpha_{1}+1}^{n_{1}}\right)\right. \\
& -2 \operatorname{Re}\left(g_{1 ; 1}^{1} \sum_{\substack{|p|=1 \\
q \geq 1}} \overline{b_{1 ; p, q}^{1}\left(w_{1}\right)^{p}}\left(\begin{array}{c}
q \\
1
\end{array}\right) x^{q-1} i\left\|w_{1}\right\|^{2 \alpha_{1}}+\cdots\right. \\
& \left.\left.+g_{1 ; 1}^{n_{1}} \sum_{\substack{|p|=1 \\
q \geq 1}} \overline{b_{1 ; p, q}^{n_{1}}\left(w_{1}\right)^{p}}\left(\begin{array}{l}
q \\
1
\end{array}\right) x^{q-1} i\left\|w_{1}\right\|^{2 \alpha_{1}}\right)\right\}=0 .
\end{aligned}
$$

Making use of (83), we obtain the relations (87) for $\ell=\alpha_{1}+1$ and (88) for $\ell=2 \alpha_{1}+1$. Analogously, by picking up the terms of total degree $4 \alpha_{1}+1$, we get the relation (88) for $\ell=2 \alpha_{1}+2$. We proceed this process degree from $4 \alpha_{1}+1$ to $6 \alpha_{1}-1$. Then we obtain (87) for $\ell=\alpha_{1}+2, \ldots, 2 \alpha_{1}$ and (88) for $\ell=2 \alpha_{1}+2, \ldots, 4 \alpha_{1}$.

Assume $a_{3} \neq 0$. The total degree $6 \alpha_{1}$ terms in $\operatorname{Im} F=\left\|G_{1}\right\|^{2 \alpha_{1}}$ satisfy the relation:

$$
\begin{aligned}
- & \sum_{q \geq 3} a_{q}\left(\begin{array}{c}
q \\
3
\end{array}\right) x^{q-3}|| w_{1}||^{6 \alpha_{1}} \\
= & \left(\left|g_{1 ; 1}^{1}\right|^{2}+\cdots+\left|g_{1 ; 1}^{n_{1}}\right|^{2}\right)^{\alpha_{1}-1} \\
& \times\left\{\left|g_{1 ; 2 \alpha_{1}+1}^{1}\right|^{2}+\cdots+\left|g_{1 ; 2 \alpha_{1}+1}^{n_{1}}\right|^{2}+2 \operatorname{Re}\left(g_{1 ; 1}^{1} \bar{g}_{1 ; 4 \alpha_{1}+1}^{1}+\cdots+g_{1 ; 1}^{n_{1}} \bar{g}_{1 ; 4 \alpha_{1}+1}^{n_{1}}\right)\right. \\
& +\left.\left|\sum_{\substack{|p|=1 \\
q \geq 1}} b_{1 ; p, q}^{1}\left(w_{1}\right)^{p}\left(\begin{array}{c}
q \\
1
\end{array}\right) x^{q-1}\right|\left|w_{1}\right|^{2 \alpha_{1}}\right|^{2}+\cdots
\end{aligned}
$$




$$
\begin{aligned}
& +\left|\sum_{\substack{|p|=1 \\
q \geq 1}} b_{1 ; p, q}^{n_{1}}\left(w_{1}\right)^{p}\left(\begin{array}{l}
q \\
1
\end{array}\right) x^{q-1}\left\|w_{1}\right\|^{2 \alpha_{1}}\right|^{2} \\
& -2 \operatorname{Re}\left(g_{1 ; 1}^{1} \sum_{\substack{|p|=1 \\
q \geq 2}} \overline{b_{1 ; p, q}^{1}\left(w_{1}\right)^{p}}\left(\begin{array}{l}
q \\
2
\end{array}\right) x^{q-2}\left\|w_{1}\right\|^{4 \alpha_{1}}+\cdots\right. \\
& \left.\left.+g_{1 ; 1}^{n_{1}} \sum_{\substack{|p|=1 \\
q \geq 2}} \overline{b_{1 ; p, q}^{n_{1}}\left(w_{1}\right)^{p}}\left(\begin{array}{l}
q \\
2
\end{array}\right) x^{q-2}\left\|w_{1}\right\|^{4 \alpha_{1}}\right)\right\} .
\end{aligned}
$$

Plug the relation (84) into the right hand side and put $x=0$. From (78), (79) and (80), we get the relation

$$
-a_{3}|| w_{1}||^{4 \alpha_{1}+2}=\left|\tilde{g}_{1 ; 2 \alpha_{1}+1}^{1}\right|^{2}+\cdots+\left|\tilde{g}_{1 ; 2 \alpha_{1}+1}^{n_{1}}\right|^{2},
$$

which means that

$$
\left(\frac{\tilde{g}_{1 ; 2 \alpha_{1}+1}^{1}}{\left(-a_{3}\right)^{1 / 2}}, \ldots, \frac{\tilde{g}_{1 ; 2 \alpha_{1}+1}^{n_{1}}}{\left(-a_{3}\right)^{1 / 2}}\right): B^{m_{1}} \rightarrow B^{n_{1}}
$$

is a proper holomorphic mapping. We apply the same argument we used to conclude $M=1$ to the mapping (92). In the argument, replace $\Phi_{M}$ by the mapping (92) and calculate the rank of the Jacobian matrix of it at the origin. Then we reach the contradiction. Therefore we conclude that $a_{3}=0$.

Assume that $a_{3}=\ldots a_{q_{0}-1}=0$ and $a_{q_{0}} \neq 0$ for $q_{0} \geq 4$. From the relation (90), we obtain

$$
\begin{aligned}
& -\sum_{q \geq q_{0}} a_{q}\left(\begin{array}{l}
q \\
3
\end{array}\right) x^{q-3}|| w_{1}||^{6 \alpha_{1}} \\
& \quad=\left(\left|g_{1 ; 1}^{1}\right|^{2}+\cdots+\left|g_{1 ; 1}^{n_{1}}\right|^{2}\right)^{\alpha_{1}-1}\left(\left|g_{1 ; 2 \alpha_{1}+1}^{1}\right|^{2}+\cdots+\left|g_{1 ; 2 \alpha_{1}+1}^{n_{1}}\right|^{2}\right) .
\end{aligned}
$$

Since the lowest degree of $x$ on the right hand side of (93) is even, we may assume the following:

- $q_{0}-3=2 \tilde{q}_{0}$,

- the lowest degree of $x$ in $g_{1 ; 2 \alpha_{1}+1}^{1}, \ldots, g_{1 ; 2 \alpha_{1}+1}^{\lambda_{0}}$ are $\tilde{q}_{0}$ and that of $x$ in $g_{1 ; 2 \alpha_{1}+1}^{\lambda_{0}+1}, \ldots, g_{1 ; 2 \alpha_{1}+1}^{n_{1}}$ is greater than $\tilde{q}_{0}$.

Divide (93) by $x^{2 \tilde{q}_{0}}$ and we get

$$
-\sum_{q \geq q_{0}} a_{q}\left(\begin{array}{l}
q \\
3
\end{array}\right) x^{q-q_{0}}\left\|w_{1}\right\|^{6 \alpha_{1}}
$$




$$
=\left(\left|g_{1 ; 1}^{1}\right|^{2}+\cdots+\left|g_{1 ; 1}^{n_{1}}\right|^{2}\right)^{\alpha_{1}-1}\left(\left|\frac{g_{1 ; 2 \alpha_{1}+1}^{1}}{x \tilde{q}_{0}}\right|^{2}+\cdots+\left|\frac{g_{1 ; 2 \alpha_{1}+1}^{n_{1}}}{x \tilde{q}_{0}}\right|^{2}\right) .
$$

Taking $x=0$ in (94), we obtain

$$
-a_{q_{0}}\left(\begin{array}{c}
q_{0} \\
3
\end{array}\right)\left\|w_{1}\right\|^{4 \alpha_{1}+2}=\left|\sum_{|p|=2 \alpha_{1}+1} b_{1 ; p, \tilde{q}_{0}}^{1}\left(w_{1}\right)^{p}\right|^{2}+\cdots+\left|\sum_{|p|=2 \alpha_{1}+1} b_{1 ; p, \tilde{q}_{0}}^{\lambda_{0}}\left(w_{1}\right)^{p}\right|^{2},
$$

which means that

$$
\left(\frac{\sum_{|p|=2 \alpha_{1}+1} b_{1 ; p, \tilde{q}_{0}}^{1}\left(w_{1}\right)^{p}}{\left(-a_{q_{0}}\left(\begin{array}{c}
q_{0} \\
3
\end{array}\right)\right)^{1 / 2}}, \ldots, \frac{\sum_{|p|=2 \alpha_{1}+1} b_{1 ; p, \tilde{q}_{0}}^{\lambda_{0}}\left(w_{1}\right)^{p}}{\left(-a_{q_{0}}\left(\begin{array}{c}
q_{0} \\
3
\end{array}\right)\right)^{1 / 2}}\right): B^{m_{1}} \rightarrow B^{\lambda_{0}}
$$

is a proper holomorphic mapping. If the inequality $m_{1}>\lambda_{0}$ holds, then this mapping can not be defined as a proper holomorphic mapping. Therefore $a_{q_{0}}=0$, which is a contradiction. Assume that $m_{1} \leq \lambda_{0}$. Then we have $m_{1} \leq \lambda_{0}\left(<n_{1}\right)<2 m_{1}-1$. Therefore by the same argument which is used to get $M=1$, we must have $2 \alpha_{1}+1=1$. This is also a contradiction. In any case, there does not exist $q_{0} \geq 4$ with $a_{q_{0}} \neq 0$. Therefore we obtain $a_{q}=0$ for $q \geq 3$. Repeat to compare the terms whose total degree of $w_{1}$ and $\bar{w}_{1}$ is bigger than $6 \alpha_{1}$, we conclude that $g_{1 ; \ell}^{\lambda}=0$ for $\lambda=1, \ldots, n_{1}$ and $\ell \geq 2$. Pick up the terms whose total degree of $w_{1}$ and $\bar{w}_{1}$ is $2 \alpha_{1}$ from $\operatorname{Im} F=\left\|G_{1}\right\|^{2 \alpha_{1}}$. Then we have

$$
1+2 a_{2} x=\frac{1}{\left\|w_{1}\right\|^{2 \alpha_{1}}}\left\{\left|g_{1 ; 1}^{1}\right|^{2}+\cdots+\left|g_{1 ; 1}^{n_{1}}\right|^{2}\right\}^{\alpha_{1}} .
$$

Divide this by $x$ and take $x$ to be infinity. Then we conclude

$$
\sum_{\substack{|p|=1 \\ q \geq 1}} b_{1 ; p, q}^{\lambda}\left(w_{1}\right)^{p} x^{q}=0, \quad \lambda=1,2, \ldots, n_{1} .
$$

Therefore $G_{1}$ is normalized to be $\left(w_{1}, 0\right)$ and $a_{2}=0$. Now the expansion of $F$, as a component of the holomorphic mapping, becomes $F=a_{0} / a_{1}+z$. Apply the shift $(\tilde{z}, \tilde{w}) \mapsto\left(\tilde{z}-a_{0} / a_{1}, \tilde{w}\right)$ to $(F, G)$. Since $a_{0} / a_{1}$ is a real number, the shift is an automorphism of $\Omega(n ;(n),(\beta))$. By this automorphism, $F$ is normalized to $F=z$.

REMARK 3. From this lemma, we conclude that $\alpha_{1}=\beta_{1}$ and that $\left(F, G_{1}\right)$ is equivalent to $\left(z, w_{1}, 0\right)$. These are the conclusions of the main theorem for $N=1$. Note that $\left(F, G_{1}\right)$ and $\left(z, w_{1}, 0\right)$ are equal up to the relation (53) and the shift. This fact is used in the proof of Lemma 5.2. 


\section{The relation among the exponents and normalization of the mapping ( $N$ block case)}

We return to the general situation, namely, we assume that the number of the block of $w$ is $N$.

Lemma 5.1. Let $\sigma$ be the permutation of $N$-indices in Lemma 2.3. Then we have $\alpha_{\sigma(i)}=\beta_{i}$.

PROOF. Take any $i$ and fix it. We restrict the mapping

$$
(F, G): \Omega(m ;(m) ;(\alpha)) \rightarrow \Omega(n ;(n) ;(\beta))
$$

to the variety

$$
\bigcap_{\substack{j=1, \ldots, N \\ j \neq \sigma(i)}}\left\{w_{j}=0\right\} .
$$

Then we have a proper holomorphic mapping

$$
\left(F, 0, \ldots, 0, G_{i}, 0, \ldots, 0\right):\left\{\operatorname{Im} z>\left\|w_{\sigma(i)}\right\|^{2 \alpha_{\sigma(i)}}\right\} \rightarrow\left\{\operatorname{Im} z>\left\|w_{i}\right\|^{2 \beta_{i}}\right\} .
$$

It follows from Lemma 4.2 that we have $\alpha_{\sigma(i)}=\beta_{i}$. Since $i$ is arbitrary, the result holds.

After reordering the variables, we may assume that $\sigma$ is identity, and therefore from now on, we use $\alpha$ instead of $\beta$.

Next, we normalize $F$.

LeMmA 5.2. Let $\left(F, G_{1}, \ldots, G_{N}\right): \Omega(m ;(m) ;(\alpha)) \rightarrow \Omega(n ;(n) ;(\alpha))$ be a proper holomorphic mapping. Then there exists an automorphism $\Phi_{n}$ of $\Omega(n ;(n) ;(\alpha))$ such that $F$ can be normalized to be z by $\Phi_{n}$, namely the first component of $\Phi_{n} \circ(F, G)$ is $z$.

Proof. Since we have $\left.G_{j}\right|_{w_{j}=0}=0$, the restriction $(F, G): \Omega(m ;(m) ;(\alpha)) \rightarrow$ $\Omega(n ;(n) ;(\alpha))$ to $w_{2}=\cdots=w_{N}=0$ is a proper holomorphic mapping of one block. So, expanding the functions $F\left(z, w_{1}, 0, \ldots, 0\right), G_{1}^{\lambda}\left(z, w_{1}, 0, \ldots, 0\right)$ as (46) and (47) in the previous section, we obtain the unitary matrix $U$ as in (52). Here $\beta_{1}$ in (46) and (47) should be replaced by $\alpha_{1}$. Let us now define an automorphism $\Phi_{n}=\left(\Phi_{n}^{0}, \ldots, \Phi_{n}^{N}\right)$ of $\Omega(n ;(n) ;(\alpha))$ by

$$
\begin{aligned}
& \Phi_{n}^{0}\left(\tilde{z}, \tilde{w}_{1}, \ldots, \tilde{w}_{N}\right)=\frac{\tilde{z}}{\left(2 k \alpha_{1}+1\right) a_{2 k \alpha_{1}+1}}+a, a \in \mathbf{R} \\
& \Phi_{n}^{1}\left(\tilde{z}, \tilde{w}_{1}, \ldots, \tilde{w}_{N}\right)=\frac{1}{\left\{\left(2 k \alpha_{1}+1\right) a_{2 k \alpha_{1}+1}\right\}^{1 / 2 \alpha_{1}}}\left(\tilde{w}_{1}^{1}, \ldots, \tilde{w}_{1}^{n_{1}}\right) \bar{U}^{t}, \\
& \Phi_{n}^{j}\left(\tilde{z}, \tilde{w}_{1}, \ldots, \tilde{w}_{N}\right)=\frac{1}{\left\{\left(2 k \alpha_{1}+1\right) a_{2 k \alpha_{1}+1}\right\}^{1 / 2 \alpha_{j}}}\left(\tilde{w}_{j}^{1}, \ldots, \tilde{w}_{j}^{n_{j}}\right), \quad j=2, \ldots, N .
\end{aligned}
$$


Then, by applying the same argument as in the proof of Lemma 4.2, it can be verified that

$$
\Phi_{n} \circ(F, G)\left(z, w_{1}, 0, \ldots, 0\right)=\left(z, w_{1}, 0, \ldots, 0\right)
$$

for $\left(z, w_{1}, 0, \ldots, 0\right) \in \Omega(m ;(m) ;(\alpha))$ and a suitable $a \in \mathbf{R}$. As we saw in Lemma 2.4 , the first component of any proper holomorphic mapping from $\Omega(m ;(m) ;(\alpha))$ to $\Omega(n ;(n) ;(\alpha))$ does not depend on the variable $w=\left(w_{1}, \ldots, w_{N}\right)$. Hence we conclude that the first component, say $\widetilde{F}$, of $\Phi_{n} \circ(F, G)$ is just of the form $\widetilde{F}(z, w)=z$ on $\Omega(m ;(m) ;(\alpha))$. This proves the lemma.

From now on, we assume that $F=z$. Therefore we shall use $\left\|\left|w\|\|^{2 \alpha}=\||| G\|\right|^{2 \alpha}\right.$ instead of $\operatorname{Im} F=\||G|\|^{2 \alpha}$ on $\operatorname{Im} z=\||| w||^{2 \alpha}$.

Next, we normalize the homogeneous polynomials in $w$ of degree one in $G_{1}, \ldots, G_{N}$. We order the exponents of $\Omega(m ;(m) ;(\alpha))$ and $\Omega(n ;(n) ;(\alpha))$ as

$$
\begin{aligned}
\alpha_{1}=\cdots=\alpha_{\ell_{1}} & <\alpha_{\ell_{1}+1}=\cdots=\alpha_{\ell_{1}+\ell_{2}} \\
& <\alpha_{\ell_{1}+\ell_{2}+1}=\cdots=\alpha_{\ell_{1}+\ell_{2}+\ell_{3}} \\
& <\alpha_{\ell_{1}+\ell_{2}+\ell_{3}+1}=\cdots=\alpha_{\ell_{1}+\ell_{2}+\ell_{3}+\ell_{4}} \\
& \vdots \\
& <\alpha_{\ell_{1}+\cdots+\ell_{\lambda}+1}=\cdots=\alpha_{\ell_{1}+\cdots+\ell_{\lambda+1}} \\
& \vdots \\
& <\alpha_{\ell_{1}+\cdots+\ell_{p-1}+1}=\cdots=\alpha_{\ell_{1}+\cdots+\ell_{p}},
\end{aligned}
$$

and $\alpha_{\ell_{1}+\cdots+\ell_{\lambda}}+\gamma_{\lambda}=\alpha_{\ell_{1}+\cdots+\ell_{\lambda+1}}$. Here we assume that $N=\ell_{1}+\cdots+\ell_{p}$. Expand $G_{j}^{\lambda}$ in homogeneous polynomials of $w$ as

$$
G_{j}^{\lambda}=\sum_{\ell \geq 1} g_{j ; \ell}^{\lambda}, \quad g_{j ; \ell}^{\lambda}=\sum_{\substack{|p|=\ell \\ q \geq 0}} b_{j ; p, q}^{\lambda}(w)^{p} z^{q} .
$$

Denote by $\hat{g}_{j ; \ell}^{\lambda}$ the restriction of $g_{j ; \ell}^{\lambda}$ to $z=x$ :

$$
\hat{g}_{j ; \ell}^{\lambda}=\sum_{\substack{|p|=\ell \\ q \geq 0}} b_{j ; p, q}^{\lambda}(w)^{p} x^{q} .
$$

Let us denote $g_{j ; \ell}=\left(g_{j ; \ell}^{\prime}, g_{j ; \ell}^{\prime \prime}\right)$ for $g_{j ; \ell}^{\prime}=\left(g_{j ; \ell}^{1}, \ldots, g_{j ; \ell}^{m_{j}}\right)$ and $g_{j ; \ell}^{\prime \prime}=\left(g_{j ; \ell}^{m_{j}+1}, \ldots, g_{j ; \ell}^{n_{j}}\right)$. Pick up the terms of total degree $2 \alpha_{1}=\cdots=2 \alpha_{\ell_{1}}$ of $w$ and $\bar{w}$ from $\left\|\left.|w|\right|^{2 \alpha}=\right\||| G \|^{2 \alpha}$. They satisfy the relation

$$
\left\|w_{1}\right\|^{2 \alpha_{1}}+\cdots+\left\|w_{\ell_{1}}\right\|^{2 \alpha_{\ell_{1}}}=\left\|\hat{g}_{1 ; 1}\right\|^{2 \alpha_{1}}+\cdots+\left\|\hat{g}_{\ell_{1} ; 1}\right\|^{2 \alpha_{\ell_{1}}} .
$$


It follows from $\left.G_{j}\right|_{w_{j}=0}=0$ that $g_{j ; 1}$ is a linear mapping in $w_{j}=\left(w_{j}^{1}, \ldots, w_{j}^{m_{j}}\right)$, which implies that $\left\|\hat{g}_{j ; 1}\left(x, w_{j}\right)\right\|^{2}=\left\|w_{j}\right\|^{2}$ for any $x$. Consider two proper holomorphic mappings $\left(g_{j ; 1}^{1}, \ldots, g_{j ; 1}^{n_{j}}\right)\left(z, w_{j}\right)$ and $\left(w_{j}, 0\right)$ between $B^{m_{j}}$ and $B^{n_{j}}$. Here $\left(g_{j ; 1}^{1}, \ldots, g_{j ; 1}^{n_{j}}\right)\left(z, w_{j}\right)$ is considered as a mapping with a variable $w_{j} \in \mathbf{C}^{m_{j}}$ and with a parameter $z \in \mathbf{C}$. Then we can find the $n_{j} \times n_{j}$ unitary matrix $U_{j}(z)$ depending on $z$ such that

$$
g_{j ; 1}\left(z, w_{j}\right)=U_{j}(z)\left(\begin{array}{c}
w_{j} \\
0
\end{array}\right) .
$$

Note that since $g_{j ; 1}$ is expanded as (107), the components of $U_{j}(z)$ are holomorphic functions of $z$. Let $\Phi_{n}^{n_{j}}\left(\tilde{z}, \tilde{w}_{j}\right)$ be a mapping defined by

$$
\Phi_{n}^{n_{j}}\left(\tilde{z}, \tilde{w}_{j}\right)=U_{j}^{-1}(\tilde{z}) \tilde{w}_{j}, \quad\left(\tilde{z}, \tilde{w}_{j}\right) \in \mathbf{C} \times \mathbf{C}^{n_{j}},
$$

where $U_{j}(z)$ is a unitary matrix as in (110). Then we can calculate as follows:

$$
\Phi_{n}^{n_{j}} \circ\left(z, g_{j ; 1}^{1}\left(z, w_{j}\right), \ldots, g_{j ; 1}^{n_{j}}\left(z, w_{j}\right)\right)=\left(w_{j}, 0\right), \quad j=1, \ldots, \ell_{1} .
$$

Note that this normalization does not depend on $z$. Next lemma is used to prove Lemma 5.4 as an induction assumption.

LEMMA 5.3. There exist an automorphism $\Phi_{n}$ of $\Omega(n ;(n) ;(\alpha))$ such that

$$
\begin{aligned}
& \Phi_{n} \circ(F, G)\left(z, w_{1}, \ldots, w_{N}\right)= \\
& \left(z, w_{1}+\sum_{\ell \geq \gamma_{1}+2} g_{1 ; \ell}^{\prime}, \sum_{\ell \geq \gamma_{1}+2} g_{1 ; \ell}^{\prime \prime}, \ldots, w_{\ell_{1}}+\sum_{\ell \geq \gamma_{1}+2} g_{\ell_{1} ; \ell}^{\prime}, \sum_{\ell \geq \gamma_{1}+2} g_{\ell_{1} ; \ell}^{\prime \prime},\right. \\
& w_{\ell_{1}+1}+\sum_{\ell \geq 2} g_{\ell_{1}+1 ; \ell}^{\prime}, \sum_{\ell \geq 2} g_{\ell_{1}+1 ; \ell}^{\prime \prime}, \ldots, w_{\ell_{1}+\ell_{2}}+\sum_{\ell \geq 2} g_{\ell_{1}+\ell_{2} ; \ell}^{\prime}, \sum_{\ell \geq 2} g_{\ell_{1}+\ell_{2} ; \ell}^{\prime \prime}, \\
& \left.G_{\ell_{1}+\ell_{2}+1}, \ldots, G_{N}\right) .
\end{aligned}
$$

Proof. Note that $F$ is already normalized to be $z$. Take $\Phi_{n}(\tilde{z}, \tilde{w})=$ $\left(\tilde{z}, \Phi_{n}^{n_{1}}\left(\tilde{z}, \tilde{w}_{1}\right), \ldots, \Phi_{n}^{n_{\ell_{1}}}\left(\tilde{z}, \tilde{w}_{\ell_{1}}\right), \tilde{w}_{\ell_{1}+1}, \ldots, \tilde{w}_{N}\right)$. Here $\Phi_{n}^{n_{j}}$ is the mapping of the form (111). Note that $\Phi_{n}^{n_{j}}$ does not depend on $\tilde{z}$. Indeed, this can be seen as follows. Let $\Phi_{n}^{n_{j}}\left(\tilde{z}, \tilde{w}_{j}\right)=\left(\phi_{1}\left(\tilde{z}, \tilde{w}_{j}\right), \ldots, \phi_{n_{j}}\left(\tilde{z}, \tilde{w}_{j}\right)\right)$. Then we have

$$
\begin{aligned}
\sum_{k=1}^{n_{j}}\left|\phi_{k}\left(\tilde{z}, \tilde{w}_{j}\right)\right|^{2} & =\left\|\Phi_{n}^{n_{j}}\left(\tilde{z}, \tilde{w}_{j}\right)\right\|^{2} \\
& =\left\|U_{j}(\tilde{z})^{-1} \tilde{w}_{j}\right\|^{2} \\
& =\left\|\tilde{w}_{j}\right\|^{2}
\end{aligned}
$$


for any $\left(\tilde{z}, \tilde{w}_{j}\right)$. Differentiate (114) with respect to $\tilde{z}$ and $\overline{\tilde{z}}$ and we have

$$
\sum_{k=1}^{n_{j}}\left|\frac{\partial \phi_{k}\left(\tilde{z}, \tilde{w}_{j}\right)}{\partial \tilde{z}}\right|^{2}=0 .
$$

Since the domain is connected, $\Phi_{n}^{n_{j}}\left(\tilde{z}, \tilde{w}_{j}\right)$ does not depend on $\tilde{z}$ and therefore $\Phi_{n}$ is an automorphism of $\Omega(n ;(n) ;(\alpha))$. Denote $\Phi_{n}^{n_{j}}=U_{j}^{-1} \tilde{w}_{j}$. We calculate the terms from the second to the $\left(n_{1}+1\right)$-st components of $\Phi_{n} \circ(F, G)$ to normalize $G_{1}$.

$$
\begin{aligned}
\Phi_{n}^{n_{1}} \circ G_{1} & =U_{1}^{-1}\left(\begin{array}{c}
\sum_{\ell \geq 1} g_{1 ; \ell}^{1} \\
\vdots \\
\sum_{\ell \geq 1} g_{1 ; \ell}^{n_{1}}
\end{array}\right) \\
& =U_{1}^{-1}\left(\begin{array}{c}
g_{1 ; 1}^{1} \\
\vdots \\
g_{1 ; 1}^{n_{1}}
\end{array}\right)+\left(\text { the terms which contain } g_{1 ; \ell}, \quad \ell \geq 2\right) .
\end{aligned}
$$

Since $g_{1 ; 1}$ is a mapping of $z$ and $w_{1}$, we see that $g_{1 ; 1}(z, w)=g_{1 ; 1}\left(z, w_{1}\right)$. As we saw in (112), $G_{1}$ is normalized as follows:

$$
\begin{aligned}
\Phi_{n}^{n_{1}} \circ & G_{1}\left(z, w_{1}, \ldots, w_{N}\right) \\
& =\Phi_{n}^{n_{1}} \circ\left(g_{1 ; 1}^{1}, \ldots, g_{1 ; 1}^{n_{1}}\right)\left(z, w_{1}\right)+\left(\text { the terms which contain } g_{1 ; \ell}, \ell \geq 2\right) \\
& =\left(w_{1}^{1}, \ldots, w_{1}^{m_{1}}, 0, \ldots, 0\right)+\left(\text { the terms which contain } g_{1 ; \ell}, \quad \ell \geq 2\right) .
\end{aligned}
$$

Using $G_{2}, \ldots, G_{\ell_{1}}$ and $\Phi_{n}^{n_{2}}, \ldots, \Phi_{n}^{n_{\ell_{1}}}$ instead of $G_{1}$ and $\Phi_{n}^{n_{1}}$, we can normalize the homogeneous polynomials of degree one $g_{2 ; 1}, \ldots, g_{\ell_{1} ; 1}$ as $\left(w_{2}, 0\right), \ldots,\left(w_{\ell_{1}}, 0\right)$ respectively.

Next we normalize the homogeneous polynomials of degree one in $G_{\ell_{1}+1}, \ldots, G_{\ell_{1}+\ell_{2}}$. Assume that $\gamma_{1} \neq 1$. Since the terms of total degree $2 \alpha_{1}+2=\cdots=2 \alpha_{\ell_{1}}+2$ of $w$ and $\bar{w}$ in $\left\|\left.\left|w\left\|\left.\right|^{2 \alpha}=\right\|\right||G|\right|^{2 \alpha}\right.$ satisfy

$$
\begin{aligned}
0= & \alpha_{1}\left\|w_{1}\right\|^{2\left(\alpha_{1}-1\right)}\left\{\left\|\hat{g}_{1 ; 2}\right\|^{2}+<w_{1}, \hat{g}_{1 ; 3}^{\prime}>+\overline{<w_{1}, \hat{g}_{1 ; 3}^{\prime}>}\right\} \\
& +\alpha_{1}\left(\alpha_{1}-1\right)\left\|w_{1}\right\|^{2\left(\alpha_{1}-2\right)}\left\{<w_{1}, \hat{g}_{1 ; 2}^{\prime}>+\overline{<w_{1}, \hat{g}_{1 ; 2}^{\prime}>}\right\}^{2} \\
& +\ldots \\
& +\alpha_{\ell_{1}}\left\|w_{\ell_{1}}\right\|^{2\left(\alpha_{\ell_{1}}-1\right)}\left\{\left\|\hat{g}_{\ell_{1} ; 2}\right\|^{2}+<w_{\ell_{1}}, \hat{g}_{\ell_{1} ; 3}^{\prime}>+\overline{<w_{\ell_{1}}, \hat{g}_{\ell_{1} ; 3}^{\prime}>}\right\} \\
& +\alpha_{\ell_{1}}\left(\alpha_{\ell_{1}}-1\right)\left\|w_{\ell_{1}}\right\|^{2\left(\alpha_{\ell_{1}}-2\right)}\left\{<w_{\ell_{1}}, \hat{g}_{\ell_{1} ; 2}^{\prime}>+\overline{<w_{\ell_{1}}, \hat{g}_{\ell_{1} ; 2}^{\prime}>}\right\}^{2},
\end{aligned}
$$

we get $\hat{g}_{1 ; 2}=\cdots=\hat{g}_{\ell_{1} ; 2}=0$, or equivalently, $g_{1 ; 2}=\cdots=g_{\ell_{1} ; 2}=0$. By picking up the terms of total degrees from $2 \alpha_{1}+4=\cdots=2 \alpha_{\ell_{1}}+4$ to $2 \alpha_{1}+2\left(\gamma_{1}-1\right)=\cdots=$ 
$2 \alpha_{\ell_{1}}+2\left(\gamma_{1}-1\right)$, the similar argument implies the following:

$$
\begin{gathered}
g_{1 ; 3}=\cdots=g_{1 ; \gamma_{1}}=0, \\
\vdots \\
g_{\ell_{1} ; 3}=\cdots=g_{\ell_{1} ; \gamma_{1}}=0 .
\end{gathered}
$$

Plug them into $\|\mid w\|^{2 \alpha}=\|\| G\|\|^{2 \alpha}$ and pick up the terms of total degree $2 \alpha_{1}+2 \gamma_{1}=\cdots=$ $2 \alpha_{\ell_{1}}+2 \gamma_{1}=2 \alpha_{\ell_{1}+1}=\cdots=2 \alpha_{\ell_{1}+\ell_{2}}$. Then we get the relation:

$$
\begin{aligned}
\left\|w_{\ell_{1}+1}\right\|^{2 \alpha_{\ell_{1}+1}}+\cdots+\left\|w_{\ell_{1}+\ell_{2}}\right\|^{2 \alpha_{\ell_{1}+\ell_{2}}} & =\alpha_{1}\left\|w_{1}\right\|^{2\left(\alpha_{1}-1\right)}\left\{\left\|\hat{g}_{1 ; \gamma_{1}+1}\right\|^{2}+<w_{1}, \hat{g}_{1 ; 2 \gamma_{1}+1}^{\prime}>+\overline{<w_{1}, \hat{g}_{1 ; 2 \gamma_{1}+1}^{\prime}>}\right\} \\
& +\alpha_{1}\left(\alpha_{1}-1\right)\left\|w_{1}\right\|^{2\left(\alpha_{1}-2\right)}\left\{<w_{1}, \hat{g}_{1 ; \gamma_{1}+1}^{\prime}>+\overline{<w_{1}, \hat{g}_{1 ; \gamma_{1}+1}^{\prime}>}\right\}^{2} \\
& +\ldots \\
& +\alpha_{\ell_{1}}\left\|w_{\ell_{1}}\right\|^{2\left(\alpha_{\ell_{1}}-1\right)}\left\{\left\|\hat{g}_{\ell_{1} ; \gamma_{1}+1}\right\|^{2}+<w_{\ell_{1}}, \hat{g}_{\ell_{1} ; 2 \gamma_{1}+1}^{\prime}>+\overline{<w_{\ell_{1}}, \hat{g}_{\ell_{1} ; 2 \gamma_{1}+1}^{\prime}}>\right\} \\
& +\alpha_{\ell_{1}}\left(\alpha_{\ell_{1}}-1\right)\left\|w_{\ell_{1}}\right\|^{2\left(\alpha_{\ell_{1}}-2\right)}\left\{<w_{\ell_{1}}, \hat{g}_{\ell_{1} ; \gamma_{1}+1}^{\prime}>+\overline{<w_{\ell_{1}}, \hat{g}_{\ell_{1} ; \gamma_{1}+1}^{\prime}>}\right\}^{2} \\
& +\left\|\hat{g}_{\ell_{1}+1 ; 1}\right\|^{2 \alpha_{\ell_{1}+1}}+\cdots+\left\|\hat{g}_{\ell_{1}+\ell_{2} ; 1}\right\|^{2 \alpha_{\ell_{1}+\ell_{2}}} .
\end{aligned}
$$

Since we have $G_{j}=0$ for $w_{j}=0$, the homogeneous polynomial of degree one $\hat{g}_{j ; 1}$ is linear in $w_{j}$. By putting $w_{1}=\cdots=w_{\ell_{1}}=0$ in (121), we get

$$
\left\|w_{\ell_{1}+1}\right\|^{2 \alpha_{\ell_{1}+1}}=\left\|g_{\ell_{1}+1 ; 1}\right\|^{2 \alpha_{\ell_{1}+1}}, \ldots,\left\|w_{\ell_{1}+\ell_{2}}\right\|^{2 \alpha_{\ell_{1}+\ell_{2}}}=\left\|g_{\ell_{1}+\ell_{2} ; 1}\right\|^{2 \alpha_{\ell_{1}+\ell_{2}}}
$$

for each fixed $z$. Applying the same argument which we have used to get the normalization (112) to (122), we can normalize the homogeneous degree one parts of $G_{\ell_{1}+1}, \ldots, G_{\ell_{1}+\ell_{2}}$ to be $\left(w_{\ell_{1}+1}, 0\right), \ldots,\left(w_{\ell_{1}+\ell_{2}}, 0\right)$. From (121) and (122), we obtain $\hat{g}_{1 ; \gamma_{1}+1}=\cdots=\hat{g}_{\ell_{1} ; \gamma_{1}+1}=0$, or equivalently, $g_{1 ; \gamma_{1}+1}=\cdots=g_{\ell_{1} ; \gamma_{1}+1}=0$. Consequently, $G_{1}, \ldots, G_{\ell_{1}+\ell_{2}}$ are normalized by $\Phi_{n}^{n_{1}}, \ldots, \Phi_{n}^{n_{\ell_{1}+\ell_{2}}}$ as follows:

$$
\begin{aligned}
G_{1} & =\left(w_{1}+\sum_{\ell \geq \gamma_{1}+2} g_{1 ; \ell}^{\prime}, \sum_{\ell \geq \gamma_{1}+2} g_{1 ; \ell}^{\prime \prime}\right), \\
& \vdots \\
G_{\ell_{1}} & =\left(w_{\ell_{1}}+\sum_{\ell \geq \gamma_{1}+2} g_{\ell_{1} ; \ell}^{\prime}, \sum_{\ell \geq \gamma_{1}+2} g_{\ell_{1} ; \ell}^{\prime \prime}\right), \\
G_{\ell_{1}+1} & =\left(w_{\ell_{1}+1}+\sum_{\ell \geq 2} g_{\ell_{1}+1 ; \ell}^{\prime}, \sum_{\ell \geq 2} g_{\ell_{1}+1 ; \ell}^{\prime \prime}\right),
\end{aligned}
$$




$$
G_{\ell_{1}+\ell_{2}}=\left(w_{\ell_{1}+\ell_{2}}+\sum_{\ell \geq 2} g_{\ell_{1}+\ell_{2} ; \ell}^{\prime}, \sum_{\ell \geq 2} g_{\ell_{1}+\ell_{2} ; \ell}^{\prime \prime}\right) .
$$

In the case of $\gamma_{1}=1$, we obtain (121) with $\gamma_{1}=1$ instead of (118). We proceed the same argument to get (123), (124), (125) and (126) with $\gamma_{1}=1$.

As the following lemma shows, we can proceed this process to normalize the homogeneous polynomials of degree one in $G$.

LEMMA 5.4. There exists an automorphism $\Phi_{n}$ of $\Omega(n ;(n) ;(\alpha))$ such that

$$
\begin{aligned}
\Phi_{n} \circ(F, G)(z, w)=\left(z, w_{1}+\sum_{\ell \geq \gamma_{1}+\cdots+\gamma_{p-1}+2} g_{1 ; \ell}^{\prime}, \sum_{\ell \geq \gamma_{1}+\cdots+\gamma_{p-1}+2} g_{1 ; \ell}^{\prime \prime}, \ldots,\right. \\
w_{\ell_{1}}+\sum_{\ell \geq \gamma_{1}+\cdots+\gamma_{p-1}+2} g_{\ell_{1} ; \ell}^{\prime}, \sum_{\ell \geq \gamma_{1}+\cdots+\gamma_{p-1}+2} g_{\ell_{1} ; \ell}^{\prime \prime}, \ldots, \\
\\
w_{\ell_{1}+\cdots+\ell_{p-1}+1}+\sum_{\ell \geq 2} g_{\ell_{1}+\cdots+\ell_{p-1}+1 ; \ell}^{\prime}, \sum_{\ell \geq 2} g_{\ell_{1}+\cdots+\ell_{p-1}+1 ; \ell}^{\prime \prime}, \ldots, \\
\left.w_{\ell_{1}+\cdots+\ell_{p}}+\sum_{\ell \geq 2} g_{\ell_{1}+\cdots+\ell_{p} ; \ell}^{\prime}, \sum_{\ell \geq 2} g_{\ell_{1}+\cdots+\ell_{p} ; \ell}^{\prime \prime}\right) .
\end{aligned}
$$

PROOF. We prove the following claim by induction, which completes the proof of this lemma.

Claim 4. For any $\lambda$, we have

$$
\begin{aligned}
\Phi_{n} \circ(F, G)(z, w) & \left(z, w_{1}+\sum_{\ell \geq \gamma_{1}+\cdots+\gamma_{\lambda-1}+2} g_{1 ; \ell}^{\prime}, \sum_{\ell \geq \gamma_{1}+\cdots+\gamma_{\lambda-1}+2} g_{1 ; \ell}^{\prime \prime}, \ldots,\right. \\
& w_{\ell_{1}}+\sum_{\ell \geq \gamma_{1}+\cdots+\gamma_{\lambda-1}+2} g_{\ell_{1} ; \ell}^{\prime}, \sum_{\ell \geq \gamma_{1}+\cdots+\gamma_{\lambda-1}+2} g_{\ell_{1} ; \ell}^{\prime \prime}, \ldots, \\
& w_{\ell_{1}+1}+\sum_{\ell \geq \gamma_{2}+\cdots+\gamma_{\lambda-1}+2} g_{\ell_{1}+1 ; \ell}^{\prime}, \sum_{\ell \geq \gamma_{2}+\cdots+\gamma_{\lambda-1}+2} g_{\ell_{1}+1 ; \ell}^{\prime \prime}, \ldots, \\
& w_{\ell_{1}+\ell_{2}}+\sum_{\ell \geq \gamma_{2}+\cdots+\gamma_{\lambda-1}+2} g_{\ell_{1}+\ell_{2} ; \ell}^{\prime}, \sum_{\ell \geq \gamma_{2}+\cdots+\gamma_{\lambda-1}+2} g_{\ell_{1}+\ell_{2} ; \ell}^{\prime \prime}, \ldots, \\
& w_{\ell_{1}+\cdots+\ell_{\lambda-1}+1}+\sum_{\ell \geq 2} g_{\ell_{1}+\cdots+\ell_{\lambda-1}+1 ; \ell}^{\prime}, \sum_{\ell \geq 2} g_{\ell_{1}+\cdots+\ell_{\lambda-1}+1 ; \ell}^{\prime \prime}, \ldots, \\
& w_{\ell_{1}+\cdots+\ell_{\lambda}}+\sum_{\ell \geq 2} g_{\ell_{1}+\cdots+\ell_{\lambda} ; \ell}^{\prime}, \sum_{\ell \geq 2} g_{\ell_{1}+\cdots+\ell_{\lambda} ; \ell}^{\prime \prime},
\end{aligned}
$$




$$
\left.G_{\ell_{1}+\cdots+\ell_{\lambda}+1}, \ldots, G_{\ell_{1}+\cdots+\ell_{p}}\right)
$$

Since we classify $(F, G)$ up to automorphisms, we sometimes use the notation $(F, G)$ instead of $\Phi_{n} \circ(F, G)$ in the proof. By Lemma 5.3, Claim 4 holds for $\lambda=2$. Assume that Claim 4 holds for $\lambda$. Assume that $\gamma_{\lambda} \neq 1$. Plug (128) into $\||w|\|^{2 \alpha}=\||| G||^{2 \alpha}$ and pick up the terms of total degree

$$
\begin{aligned}
2 \alpha_{1}+ & 2\left(\gamma_{1}+\cdots+\gamma_{\lambda-1}\right)+2=\cdots=2 \alpha_{\ell_{1}}+2\left(\gamma_{1}+\cdots+\gamma_{\lambda-1}\right)+2 \\
& =2 \alpha_{\ell_{1}+1}+2\left(\gamma_{2}+\cdots+\gamma_{\lambda-1}\right)+2=\cdots=2 \alpha_{\ell_{1}+\ell_{2}}+2\left(\gamma_{2}+\cdots+\gamma_{\lambda-1}\right)+2 \\
& =\cdots=2 \alpha_{\ell_{1}+\cdots+\ell_{\lambda-1}+1}+2=\cdots=2 \alpha_{\ell_{1}+\cdots+\ell_{\lambda}}+2 .
\end{aligned}
$$

Then we get the following $\lambda$ relations:

$$
\begin{aligned}
& \alpha_{1}\left\|w_{1}\right\|^{2\left(\alpha_{1}-1\right)}\left\{\left\|\hat{g}_{1 ; \gamma_{1}+\cdots+\gamma_{\lambda-1}+2}\right\|^{2}\right. \\
& \left.+<w_{1}, \hat{g}_{1 ; 2\left(\gamma_{1}+\cdots+\gamma_{\lambda-1}\right)+3}^{\prime}>+\overline{<w_{1}, \hat{g}_{1 ; 2\left(\gamma_{1}+\cdots+\gamma_{\lambda-1}\right)+3}^{\prime}>}\right\} \\
& +\alpha_{1}\left(\alpha_{1}-1\right)\left\|w_{1}\right\|^{2\left(\alpha_{1}-2\right)} \\
& \left\{<w_{1}, \hat{g}_{1 ; \gamma_{1}+\cdots+\gamma_{\lambda-1}+2}^{\prime}>+\overline{<w_{1}, \hat{g}_{1 ; \gamma_{1}+\cdots+\gamma_{\lambda-1}+2}^{\prime}>}\right\}^{2} \\
& +\ldots \\
& +\alpha_{\ell_{1}}\left\|w_{\ell_{1}}\right\|^{2\left(\alpha_{\ell_{1}}-1\right)}\left\{\left\|\hat{g}_{\ell_{1} ; \gamma_{1}+\cdots+\gamma_{\lambda-1}+2}\right\|^{2}\right. \\
& \left.+<w_{\ell_{1}}, \hat{g}_{\ell_{1} ; 2\left(\gamma_{1}+\cdots+\gamma_{\lambda-1}\right)+3}^{\prime}>+\overline{<w_{\ell_{1}}, \hat{g}_{\ell_{1} ; 2\left(\gamma_{1}+\cdots+\gamma_{\lambda-1}\right)+3}^{\prime}>}\right\} \\
& +\alpha_{\ell_{1}}\left(\alpha_{\ell_{1}}-1\right)\left\|w_{\ell_{1}}\right\|^{2\left(\alpha_{\ell_{1}}-2\right)} \\
& \left\{<w_{\ell_{1}}, \hat{g}_{\ell_{1} ; \gamma_{1}+\cdots+\gamma_{\lambda-1}+2}^{\prime}>+\overline{<w_{\ell_{1}}, \hat{g}_{\ell_{1} ; \gamma_{1}+\cdots+\gamma_{\lambda-1}+2}^{\prime}>}\right\}^{2}=0, \\
& \alpha_{\ell_{1}+\cdots+\ell_{\lambda-1}+1}\left\|w_{\ell_{1}+\cdots+\ell_{\lambda-1}+1}\right\|^{2\left(\alpha_{\ell_{1}+\cdots+\ell_{\lambda-1}+1}-1\right)} \\
& \left\{\left\|\hat{g}_{\ell_{1}+\cdots+\ell_{\lambda-1}+1 ; 2}\right\|^{2}+<w_{\ell_{1}+\cdots+\ell_{\lambda-1}+1}, \hat{g}_{\ell_{1}+\cdots+\ell_{\lambda-1}+1 ; 3}^{\prime}>\right. \\
& \left.+\overline{<w_{\ell}+\cdots+\ell_{\lambda-1}+1}, \hat{g}_{\ell_{1}+\cdots+\ell_{\lambda-1}+1 ; 3}^{\prime}>\right\} \\
& +\alpha_{\ell_{1}+\cdots+\ell_{\lambda-1}+1}\left(\alpha_{\ell_{1}+\cdots+\ell_{\lambda-1}+1}-1\right)\left\|w_{\ell_{1}+\cdots+\ell_{\lambda-1}+1}\right\|^{2\left(\alpha_{\ell_{1}+\cdots+\ell_{\lambda-1}+1}-2\right)} \\
& \left\{<w_{\ell_{1}+\cdots+\ell_{\lambda-1}+1}, \hat{g}_{\ell_{1}+\cdots+\ell_{\lambda-1}+1 ; 2}^{\prime}>\right. \\
& \left.+\overline{<w_{\ell_{1}+\cdots+\ell_{\lambda-1}+1}, \hat{g}_{\ell_{1}+\cdots+\ell_{\lambda-1}+1 ; 2}^{\prime}>}\right\}^{2}
\end{aligned}
$$




$$
\begin{aligned}
& +\ldots \\
& +\alpha_{\ell_{1}+\cdots+\ell_{\lambda}}\left\|w_{\ell_{1}+\cdots+\ell_{\lambda}}\right\|^{2\left(\alpha_{\left.\ell_{1}+\cdots+\ell_{\lambda}-1\right)}\right.} \\
& \quad\left\{\left\|\hat{g}_{\ell_{1}+\cdots+\ell_{\lambda} ; 2}\right\|^{2}+<w_{\ell_{1}+\cdots+\ell_{\lambda}}, \hat{g}_{\ell_{1}+\cdots+\ell_{\lambda} ; 3}^{\prime}>\right. \\
& \left.+\overline{<w_{\ell_{1}+\cdots+\ell_{\lambda}}, \hat{g}_{\ell_{1}+\cdots+\ell_{\lambda} ; 3}^{\prime}>}\right\} \\
& +\alpha_{\ell_{1}+\cdots+\ell_{\lambda}}\left(\alpha_{\ell_{1}+\cdots+\ell_{\lambda}}-1\right)\left\|w_{\ell_{1}+\cdots+\ell_{\lambda}}\right\|^{2\left(\alpha_{\left.\ell_{1}+\cdots+\ell_{\lambda}-2\right)}\right.} \\
& \quad\left\{<w_{\ell_{1}+\cdots+\ell_{\lambda}}, \hat{g}_{\ell_{1}+\cdots+\ell_{\lambda} ; 2}^{\prime}>+\overline{<w_{\ell_{1}+\cdots+\ell_{\lambda}}, \hat{g}_{\ell_{1}+\cdots+\ell_{\lambda} ; 2}^{\prime}>}\right\}^{2}=0 .
\end{aligned}
$$

These relations imply that

$$
\begin{aligned}
& g_{1 ; \gamma_{1}+\cdots+\gamma_{\lambda-1}+2}=\cdots=g_{\ell_{1} ; \gamma_{1}+\cdots+\gamma_{\lambda-1}+2}=0, \\
& \vdots \\
& g_{\ell_{1}+\cdots+\ell_{\lambda-1}+1 ; 2}=\cdots=g_{\ell_{1}+\cdots+\ell_{\lambda} ; 2}=0 .
\end{aligned}
$$

Plugging them into (128) and comparing the terms in the both sides of $\left\||w|||^{2 \alpha}=\right\||| G \mid \|^{2 \alpha}$ of total degrees from

$$
\begin{aligned}
2 \alpha_{1}+ & 2\left(\gamma_{1}+\cdots+\gamma_{\lambda-1}\right)+4=\cdots=2 \alpha_{\ell_{1}}+2\left(\gamma_{1}+\cdots+\gamma_{\lambda-1}\right)+4 \\
& =2 \alpha_{\ell_{1}+1}+2\left(\gamma_{2}+\cdots+\gamma_{\lambda-1}\right)+4=\cdots=2 \alpha_{l \ell_{1}+\ell_{2}}+2\left(\gamma_{2}+\cdots+\gamma_{\lambda-1}\right)+4 \\
& =\cdots=2 \alpha_{\ell_{1}+\cdots+\ell_{\lambda-1}+1}+4=\cdots=2 \alpha_{\ell_{1}+\cdots+\ell_{\lambda}}+4
\end{aligned}
$$

to

$$
\begin{aligned}
2 \alpha_{1}+ & 2\left(\gamma_{1}+\cdots+\gamma_{\lambda}\right)-2=\cdots=2 \alpha_{\ell_{1}}+2\left(\gamma_{1}+\cdots+\gamma_{\lambda}\right)-2 \\
& =2 \alpha_{\ell_{1}+1}+2\left(\gamma_{2}+\cdots+\gamma_{\lambda}\right)-2=\cdots=2 \alpha_{\ell_{1}+\ell_{2}}+2\left(\gamma_{2}+\cdots+\gamma_{\lambda}\right)-2 \\
& =\cdots=2 \alpha_{\ell_{1}+\cdots+\ell_{\lambda-1}+1}+2 \gamma_{\lambda}-2=\cdots=2 \alpha_{\ell_{1}+\cdots+\ell_{\lambda}}+2 \gamma_{\lambda}-2,
\end{aligned}
$$

we obtain

$$
\begin{aligned}
& g_{1 ; \gamma_{1}+\cdots+\gamma_{\lambda-1}+3}=\cdots=g_{1 ; \gamma_{1}+\cdots+\gamma_{\lambda}}=0, \\
& \vdots \\
& g_{\ell_{1} ; \gamma_{1}+\cdots+\gamma_{\lambda-1}+3}=\cdots=g_{\ell_{1} ; \gamma_{1}+\cdots+\gamma_{\lambda}}=0, \\
& \quad \vdots \\
& g_{\ell_{1}+\cdots+\ell_{\lambda-1}+1 ; 3}=\cdots=g_{\ell_{1}+\cdots+\ell_{\lambda-1}+1 ; \gamma_{\lambda}}=0, \\
& \quad \vdots \\
& g_{\ell_{1}+\cdots+\ell_{\lambda} ; 3}=\cdots=g_{\ell_{1}+\cdots+\ell_{\lambda} ; \gamma_{\lambda}}=0
\end{aligned}
$$


by the same argument as to get (132) and (133). Next pick up the terms of total degree

$$
\begin{aligned}
2 \alpha_{1}+ & 2\left(\gamma_{1}+\cdots+\gamma_{\lambda}\right)=\cdots=2 \alpha_{\ell_{1}}+2\left(\gamma_{1}+\cdots+\gamma_{\lambda}\right) \\
& =2 \alpha_{\ell_{1}+1}+2\left(\gamma_{2}+\cdots+\gamma_{\lambda}\right)=\cdots=2 \alpha_{\ell_{1}+\ell_{2}}+2\left(\gamma_{2}+\cdots+\gamma_{\lambda}\right) \\
& =\cdots=2 \alpha_{\ell_{1}+\cdots+\ell_{\lambda-1}+1}+2 \gamma_{\lambda}=\cdots=2 \alpha_{\ell_{1}+\cdots+\ell_{\lambda}}+2 \gamma_{\lambda} \\
& =2 \alpha_{\ell_{1}+\cdots+\ell_{\lambda}+1}=\cdots=2 \alpha_{\ell_{1}+\cdots+\ell_{\lambda+1}} .
\end{aligned}
$$

We get the analogous, but containing more terms, equation (121) and obtain

$$
\begin{aligned}
& g_{1 ; \gamma_{1}+\cdots+\gamma_{\lambda}+1}=\cdots=g_{\ell_{1} ; \gamma_{1}+\cdots+\gamma_{\lambda}+1}=0, \\
& \vdots \\
& g_{\ell_{1}+\cdots+\ell_{\lambda-1}+1 ; \gamma_{\lambda}+1}=\cdots=g_{\ell_{1}+\cdots+\ell_{\lambda} ; \gamma_{\lambda}+1}=0,
\end{aligned}
$$

and a proper holomorphic mapping

$$
\left(g_{j ; 1}^{1}, \ldots, g_{j ; 1}^{n_{j}}\right): B^{m_{j}} \rightarrow B^{n_{j}}, \quad j=\ell_{1}+\cdots+\ell_{\lambda}+1, \ldots, \ell_{1}+\cdots+\ell_{\lambda+1}
$$

for fixed $z$. By the same argument as in Lemma 5.3, the homogeneous polynomials of degree one in $G_{\ell_{1}+\cdots+\ell_{\lambda}+1}, \ldots, G_{\ell_{1}+\cdots+\ell_{\lambda+1}}$ are normalized to be

$$
\begin{gathered}
\left(g_{\ell_{1}+\cdots+\ell_{\lambda}+1 ; 1}^{\prime}, g_{\ell_{1}+\cdots+\ell_{\lambda}+1 ; 1}^{\prime \prime}\right)=\left(w_{\ell_{1}+\cdots+\ell_{\lambda}+1}, 0\right), \\
\vdots \\
\left(g_{\ell_{1}+\cdots+\ell_{\lambda+1} ; 1}^{\prime}, g_{\ell_{1}+\cdots+\ell_{\lambda+1} ; 1}^{\prime \prime}\right)=\left(w_{\ell_{1}+\cdots+\ell_{\lambda+1}}, 0\right) .
\end{gathered}
$$

This means that (128) holds for $\lambda+1$.

In the case of $\gamma_{\lambda}=1$, we can proceed the same argument to get (141), (142) with $\gamma_{\lambda}=1$ and (144) and (145).

Taking $\lambda=p$, we complete the proof.

LEMMA 5.5. All $g_{j ; l}^{\prime}$ and $g_{j ; l}^{\prime \prime}$ appearing in (127) vanish.

Proof. Among the degrees of $w_{1}, \ldots, w_{N}$ appearing on the left hand side of $\||w|\|^{2 \alpha}=\||| G \mid\|^{2 \alpha}$, the highest degree is $2 \alpha_{\ell_{1}+\cdots+\ell_{p-1}+1}=\cdots=2 \alpha_{\ell_{1}+\cdots+\ell_{p}}$. By picking up the terms of degree bigger than them from $\||G|||^{2 \alpha}$, we conclude the result. theorem.

Now we have proved that $g_{j ; \ell}=0$ for $\ell \geq 2$; thereby, completing the proof of the main

\section{The mappings between generalized pseudoellipsoids and assumptions on di-} mensions

6.1. The mappings between generalized pseudoellipsoids. In this sub-section, we compare Kodama's result [7] with our result. According to the proof of the main theorem, any 
proper holomorphic mapping

$$
\left(\mathcal{F}, \mathcal{G}_{1}, \ldots, \mathcal{G}_{N}\right): E(m ;(m) ;(\alpha)) \rightarrow E(n ;(n) ;(\beta))
$$

is equivalent to $\left(\widetilde{\mathcal{F}}, \widetilde{\mathcal{G}_{1}}, \ldots, \widetilde{\mathcal{G}_{N}}\right)$ of the form

$$
\widetilde{\mathcal{F}}\left(z, w_{1}, \ldots, w_{N}\right)=z, \quad \widetilde{\mathcal{G}}_{j}\left(z, w_{1}, \ldots, w_{N}\right)=\left(w_{\sigma(j)}, 0\right), \quad 1 \leq j \leq N
$$

via the transformations $\Psi_{1}, \Psi_{2}$ (see (3)) and the following:

$$
\begin{aligned}
& \phi_{a}:(\tilde{z}, \tilde{w}) \mapsto(\tilde{z}+a, \tilde{w}), \\
& \phi_{\widetilde{U}}:(\tilde{z}, \tilde{w}) \mapsto\left(\tilde{z}, \widetilde{U}_{1} \tilde{w}_{1}, \ldots, \widetilde{U}_{N} \tilde{w}_{N}\right), \\
& \phi_{A}:(\tilde{z}, \tilde{w}) \mapsto\left(A \tilde{z}, A^{\frac{1}{2 \alpha_{1}}} \tilde{w}_{1}, \ldots, A^{\frac{1}{2 \alpha_{N}}} \tilde{w}_{N}\right), \\
& \phi_{\sigma}:(\tilde{z}, \tilde{w}) \mapsto\left(\tilde{z}, \tilde{w}_{\sigma(1)}, \ldots, \tilde{w}_{\sigma(N)}\right),
\end{aligned}
$$

for $a, A \in \mathbf{R}, \widetilde{U}_{1}, \ldots, \widetilde{U}_{N}$ being unitary and $\sigma$ being a permutation of $\{1, \ldots, N\}$. Therefore $\left(\mathcal{F}, \mathcal{G}_{1}, \ldots, \mathcal{G}_{N}\right)$ is a composite mapping of these mappings.

$$
\begin{aligned}
\left(\mathcal{F}, \mathcal{G}_{1}, \ldots, \mathcal{G}_{N}\right) & \\
= & \Psi_{2} \circ \phi_{\sigma} \circ \phi_{A} \circ \phi_{\widetilde{U}} \circ \phi_{a} \circ\left(z, w_{1}, 0, w_{2}, 0, \ldots, w_{N}, 0\right) \circ \Psi_{1}^{-1}(z, w) \\
= & \left(\frac{z(i+A i-a A)+(i-A i-a A)}{z(i-A i+a A)+(i+A i+a A)},\right. \\
& \left(\frac{-4 A}{\{z(i-A i+a A)+(i+A i+a A)\}^{2}}\right)^{\frac{1}{2 \alpha_{\sigma(1)}}} \widetilde{U}_{\sigma(1)}\left(w_{\sigma(1)}, 0\right), \ldots, \\
& \left.\left(\frac{-4 A}{\{z(i-A i+a A)+(i+A i+a A)\}^{2}}\right)^{\frac{1}{2 \alpha_{\sigma(N)}}} \widetilde{U}_{\sigma(N)}\left(w_{\sigma(N)}, 0\right)\right) .
\end{aligned}
$$

Put

$$
c=-\frac{-i+A i+a A}{-i-A i+a A}, \quad e^{i \theta}=\frac{-i-A i+a A}{i+A i+a A}, \quad U_{j}=\exp \left(i \frac{\theta-\pi}{2 \alpha_{j}}\right) \widetilde{U}_{j}
$$

then, by calculation, the mapping (152) is re-written as

$$
\begin{aligned}
\left(\mathcal{F}, \mathcal{G}_{1}, \ldots, \mathcal{G}_{N}\right)=\left(e^{i \theta} \frac{z-c}{\bar{c} z-1},\right. & \left\{\frac{1-|c|^{2}}{(1-\bar{c} z)^{2}}\right\}^{\frac{1}{2 \alpha_{\sigma(1)}}} U_{\sigma(1)}\left(w_{\sigma(1)}, 0\right), \ldots, \\
& \left.\left\{\frac{1-|c|^{2}}{(1-\bar{c} z)^{2}}\right\}^{\frac{1}{2 \alpha_{\sigma(N)}}} U_{\sigma(N)}\left(w_{\sigma(N)}, 0\right)\right) .
\end{aligned}
$$

From this expression, we conclude that if $m_{\sigma(j)}=n_{j}$ and a proper holomorphic mapping

$$
\left(\mathcal{F}, \mathcal{G}_{1}, \ldots, \mathcal{G}_{N}\right): E(m ;(m) ;(\alpha)) \rightarrow E(n ;(n) ;(\beta))
$$


exists, then the equality $\alpha_{\sigma(j)}=\beta_{j}$ holds and any such mapping is a biholomorphic mapping. That is, any proper self mapping of $E(m ;(m) ;(\alpha))$ is a holomorphic automorphism.

A. Kodama [7] obtained the holomorphic automorphism group of a generalized complex ellipsoid

$$
\begin{aligned}
& E\left(n_{0}, \ldots, n_{K} ; p_{0}, \ldots, p_{K}\right) \\
& \quad=\left\{\left(z_{0}, \ldots, z_{K}\right) \in \mathbf{C}^{n_{0}} \times \cdots \times \mathbf{C}^{n_{K}} ; \sum_{k=0}^{K}\left\|z_{k}\right\|^{2 p_{k}}<1\right\},
\end{aligned}
$$

where $n_{0}, \ldots, n_{K}$ are positive integers and $p_{0}, \ldots, p_{K}$ are positive real numbers. Let $n_{0}+$ $\cdots+n_{K}=N$. The special case of Kodama's domain $E\left(1, n_{1}, \ldots, n_{K} ; 1, p_{1}, \ldots, p_{K}\right)$ for positive integers $p_{1}, \ldots, p_{K}$ coincides with our domain $E\left(N ; n_{1}, \ldots, n_{K} ; p_{1}, \ldots, p_{K}\right)$ and, in this setting, our mapping (154) with $m_{\sigma(j)}=n_{j}$ coincides with the Kodama's result [7].

6.2. Assumptions on dimensions. In this sub-section, we discuss about the assumptions on dimensions. About the inequality $m_{\sigma(j)} \leq n_{j}<2 m_{\sigma(j)}-1$ in (2) of the main theorem, we used it to get $M=1, a_{3}=0$ and non-existence of $q_{0}$ in Lemma 4.2. The key of the argument is to prove the injectivity of $\Phi_{M}$ in (75). Therefore if we know that any proper homogeneous holomorphic mapping $f: B^{m} \rightarrow B^{n}$ for any $m, n$ with $m<n$ is injective, our argument may work. This leads to the conjecture.

CONJECTURE 6.1. Let $m$ and $n$ be integers with $2<m<n$. If any proper homogeneous holomorphic mapping $f: B^{m} \rightarrow B^{n}$ is injective, then the conclusion (2) of the main theorem holds without the assumption $m_{\sigma(j)} \leq n_{j}<2 m_{\sigma(j)}-1$.

This conjecture excludes the case $m=2$ and $n=3$. In the main theorem, we assume that $m_{j}>2$ and $n_{j}>3$ for all $j$. As stated in the introduction, any proper holomorphic mapping between balls of dimensions two and three which is twice differentiable up to the boundary is equivalent to one of the following four mappings:

$$
(z, w) \mapsto(z, w, 0), \quad\left(z^{2}, \sqrt{2} z w, w^{2}\right), \quad\left(z, z w, w^{2}\right), \quad\left(z^{3}, \sqrt{3} z w, w^{3}\right) .
$$

Note that while the first two mappings satisfy

$$
\|(z, w, 0)\|^{2}=\|(z, w)\|^{2}, \quad\left\|\left(z^{2}, \sqrt{2} z w, w^{2}\right)\right\|^{2}=\|(z, w)\|^{4},
$$

the last two mappings do not, namely, there do not exist positive integers $\alpha$ and $\beta$ such that

$$
\left\|\left(z, z w, w^{2}\right)\right\|^{2}=\|(z, w)\|^{2 \alpha}, \quad\left\|\left(z^{3}, \sqrt{3} z w, w^{3}\right)\right\|^{2}=\|(z, w)\|^{2 \beta} .
$$

Therefore we have the conjecture.

CONJECTURE 6.2. In the main theorem, if there exist $j$ such that $n_{j}=3$ and $m_{\sigma(j)}=$ 2, say, $j=1, \ldots, j_{0}$, then $\mathcal{G}_{1}, \ldots, \mathcal{G}_{j_{0}}$ are equivalent to one of two:

$$
\left(w_{j}^{1}, w_{j}^{2}, 0\right), \quad\left(\left(w_{j}^{1}\right)^{2}, \sqrt{2} w_{j}^{1} w_{j}^{2},\left(w_{j}^{2}\right)^{2}\right) .
$$


If $\mathcal{G}_{j}$ is equivalent to the former, then $\alpha_{\sigma(j)}=\beta_{j}$, and equivalent to the later, then $\alpha_{\sigma(j)}=$ $2 \beta_{j}$.

ACKNOWLEDGMENT. The author thanks the referee for many helpful comments and suggestions. Besides them, the referee pointed out some mistakes in Lemma 2.2. Referee's comments and advices simplified the argument and made this article easy to read.

\section{References}

[ 1 ] P. Ebenfelt. and D. N. Son, Holomorphic mappings between pseudoellipsoids in different dimension, Methods Appl. Anal. 21, 365-377 (2014).

[2] J. FARAN, Maps from the two-ball to the three-ball, Invent. Math. 68 (1982), 441-475.

[ 3 ] J. FARAN, On the linearity of proper maps between balls in the low codimensional case, J. Diff. Geom. 24 (1986), 15-17.

[ 4 ] X. HUANG, On the linearity problem for proper holomorphic maps between balls in complex spaces of different dimensions, J. Diff. Geom. 51 (1999), 13-33.

[ 5 ] X. HuAng, S. Ji and D. XU, A new gap phenomenon for proper holomorphic mappings from $B^{n}$ into $B^{N}$, Math. Res. Lett. 13 (2006), no. 4, 515-529.

[6] X. Huang, S. Ji and W. Yin, A Survey on the Recent Progress of Some Problems in Several Complex Variables, ICCM 2007, Vol. I, 563-575.

[ 7 ] A. Kodama, On the holomorphic automorphism group of a generalized complex ellipsoid, Complex Var. Elliptic Equ. 59 (2014), no. 9, 1342-1349.

[ 8 ] W. Rudin, Function Theory in the Unit Balls of $\mathbf{C}^{n}$, Springer-Verlag, New York, Heidelberg and Berlin, 1980.

[9] S. WeBster, On mapping an $n$ ball into an $n+1$ ball in complex space, Pacific J. Math. 81 (1977), $267-277$.

Present Address:

Nagano national College of Technology,

716 TOKUMA, NAGANO 381-8550, JAPAN.

e-mail: atsushi@nagano-nct.ac.jp 